\title{
Antifungal Activity of New Eugenol-Benzoxazole Hybrids against Candida spp.
}

\author{
Larissa Incerti Santos de Carvalho, ${ }^{1}$ Dalila Junqueira Alvarenga, \\ Letícia Cruz Ferreira do Carmo, ${ }^{1}$ Lucas Gomes de Oliveira, ${ }^{1}$ Naiara Chaves Silva, ${ }^{2}$ \\ Amanda Latércia Tranches Dias, ${ }^{2}$ Luiz Felipe Leomil Coelho, ${ }^{2}$ \\ Thiago Belarmino de Souza, ${ }^{3}$ Danielle Ferreira Dias, ${ }^{3}$ and Diogo Teixeira Carvalho ${ }^{1}$ \\ ${ }^{1}$ Department of Foods and Drugs, Faculty of Pharmaceutical Sciences, Federal University of Alfenas, \\ 37130-001 Alfenas, MG, Brazil \\ ${ }^{2}$ Department of Microbiology and Immunology, Institute of Biomedical Sciences, Federal University of Alfenas, \\ 37130-001 Alfenas, MG, Brazil \\ ${ }^{3}$ Institute of Chemistry, Federal University of Alfenas, 37130-001 Alfenas, MG, Brazil
}

Correspondence should be addressed to Diogo Teixeira Carvalho; diogotcarv@gmail.com

Received 8 March 2017; Accepted 8 June 2017; Published 24 July 2017

Academic Editor: Ponnurengam Malliappan Sivakumar

Copyright (C) 2017 Larissa Incerti Santos de Carvalho et al. This is an open access article distributed under the Creative Commons Attribution License, which permits unrestricted use, distribution, and reproduction in any medium, provided the original work is properly cited.

\begin{abstract}
Eugenol is a natural allylphenol responsible for a wide range of biological activities, especially antimicrobial. Benzoxazoles are heterocycles with recognized antimicrobial activities. This paper describes the design, synthesis, and the biological results for benzoxazole type derivatives of eugenol as antifungal agents. The products were obtained in good yields by a four-step synthetic sequence involving aromatic nitration, nitroreduction, amide formation, and cycle condensation. They were evaluated against species of Candida spp. in microdilution assays, and four products $\left(5 \mathrm{a}, 5 \mathrm{~b}^{\prime}, 5 \mathrm{c}\right.$, and $\left.5 \mathrm{~d}^{\prime}\right)$ were about five times more active than eugenol against C. albicans and C. glabrata. Two of them $\left(5 \mathrm{~b}^{\prime}\right.$ and $\left.5 \mathrm{~d}^{\prime}\right)$ showed good activity against C. krusei, a species which is naturally resistant to fluconazole. Furthermore, the active products were more selective than eugenol against human blood cells, showing that they are interesting substances for further optimization.
\end{abstract}

\section{Introduction}

Infectious diseases are among the leading causes of deaths annually, mainly in low income countries [1]. In this context, opportunistic fungal infections, for example, candidiasis, represent a serious medical problem, since they may affect immunocompromised patients. Candida spp. infections are usually controlled by azoles or amphotericin B-based therapies, but the increasing fungal resistance findings limit the therapeutic success and new drugs have been constantly sought [2].

Heterocyclic compounds are widely known for their distinct biological activities and many of them constitute pharmacophores in important drug molecules as macrolides, aminoglycosides and $\beta$-lactam antibiotics, azole antifungals,
HIV protease and integrase inhibitors, and natural anticancer agents [3]. Nitrogen-based heterocycles are among the most frequently used ring systems in drug discovery [4]. Benzoxazoles are nitrogen-oxygen containing heterocycles that occur in a number of natural products and are easily obtained by total synthesis. Many substances containing this cyclic moiety were described in view of their wide range of biological activities. Ertan et al. (2004) have synthesized a set of very active benzoxazoles against Bacillus subtilis, which also showed relevant antimicrobial activities against Escherichia coli and Candida albicans [5]. Potent benzoxazoles showing HIV reverse transcriptase inhibition were reported by Medebielle and colleagues and these products presented antifungal activities against Aspergillus niger and A. flavus [6]. In 2008, Jauhari and colleagues described the cytotoxic activity of 
synthetic benzoxazoles against cervical, colon, and breast adenocarcinoma cell lines [7], while Arisoy and contributors described the relevant activities of 2,5-disubstituted benzoxazoles against Candida krusei and gentamicin-resistant isolates of Pseudomonas aeruginosa [8]. Kim et al. (2010) have prepared benzoxazole amides, which presented good activity against Malassezia furfur, a pathogenic fungus, involved in fatal sepsis cases [9]. Recently, Kuroyanagi and colleagues showed the outstanding potential of some 1,3-benzoxazol-4carbonitriles against Candida strains [10].

At the same time, eugenol (1), a natural allylphenol found mainly in cloves [Eugenia caryophyllata L. Merr. \& Perry (Myrtaceae)], shows relevant pharmacological activities, especially the antibacterial and antifungal actions [11]. Some authors have described the synthesis of eugenol derivatives in order to optimize its activities. The antimicrobial actions of eugenol seem to be related to cellular membranes interferences [12-14].

Following our interest in obtaining bioactive eugenol derivatives and in face of the potential of benzoxazole derivatives as antimicrobial agents, we present herein the synthesis and anti-Candida activity of eugenol-benzoxazole based hybrids. Besides eugenol, we also employed dihydroeugenol as a starting material, in order to evaluate the influence of the allylic chain on antifungal activity.

\section{Materials and Methods}

2.1. Chemistry. Thin-layer chromatography (TLC) on silica gel-G plates (Macherey-Nagel, DC-Fertigfolien ALUGRAM $^{\circledR}$ Xtra Sil G/UV ${ }_{254}$ ) was used to monitor reactions courses. Determination of the $\mathrm{Rf}$ values for the final products was done with the same TLC plates employing hexane/ethyl acetate $(80: 20, \mathrm{v} / \mathrm{v})$ for all compounds. For column chromatography, column grade silica gel (Sorbiline; $0.040-0.063 \mathrm{~mm}$ mesh size) was employed. Melting points of the compounds were obtained on Microquímica MOAs 301 melting-point apparatus and are uncorrected. IR spectra were recorded on a Shimadzu FTIR-Affinity-1 spectrometer. NMR spectra were recorded on a Bruker AC-300 spectrometer (Rheinstetten, Germany) (300 MHz for ${ }^{1} \mathrm{H}-\mathrm{NMR}$ and $75 \mathrm{MHz}$ for ${ }^{13} \mathrm{C}$-NMR spectra) in deuterated chloroform. Chemical shifts $(\delta)$ were reported in parts per million (ppm) with reference to tetramethylsilane (TMS) as internal standard and coupling constants $(J)$ were reported in Hertz $(\mathrm{Hz})$. High-resolution mass (HRMS) spectra were obtained for the final products on a quadrupole time-of-flight instrument (micrOTOF-QII, Bruker Daltonics, Billerica, MA, USA), equipped with an ESI positive and negative ion source.

Synthesis of Nitroderivatives 2 and $\mathbf{2}^{\prime}$. To a solution of eugenol $(\mathbf{1}, 32.53 \mathrm{mmol})$ or dihydroeugenol $\left(\mathbf{1}^{\prime}, 31.26 \mathrm{mmol}\right)$ in dichloromethane $(50 \mathrm{~mL})$ were added equimolar amounts of sodium bisulfate and potassium nitrate, followed by addition of wet silica gel $(1: 1 \mathrm{w} / \mathrm{w} ; 6.5 \mathrm{~g}$ for eugenol reaction and $6.3 \mathrm{~g}$ for dihydroeugenol reaction). The reaction was kept under vigorous magnetic stirring at room temperature for 48 hours and monitored by TLC (chloroform/methanol, $9.5: 0.5, \mathrm{v} / \mathrm{v}$ ).
After completion of the reaction, the mixture was filtered off; the filtrate was dried over anhydrous sodium sulfate and concentrated under reduced pressure. The nitrocompounds $\mathbf{2}$ and $\mathbf{2}^{\prime}$ were purified by column chromatography (hexane/ethyl acetate, $9: 1, \mathrm{v} / \mathrm{v}$ ) or by multiple extractions with hexane.

4-Allyl-2-methoxy-6-nitrophenol (2). Yellow oil; 37\% yield; IR $\left(\bar{v}_{\text {máx }}\right.$, in $\left.\mathrm{cm}^{-1}\right): 3520(\mathrm{O}-\mathrm{H}), 1600,1550(\mathrm{C}=\mathrm{C}), 1515,1440$ $\left(\mathrm{NO}_{2}\right), 1270(\mathrm{C}-\mathrm{O}-\mathrm{C}) ;{ }^{1} \mathrm{H} \mathrm{NMR}\left(\delta ; \mathrm{CDCl}_{3} ; 300 \mathrm{MHz}\right): 10.6(\mathrm{~s}$, $1 \mathrm{H}, \mathrm{O}-\underline{\mathrm{H}}), 7.4(\mathrm{~d}, 1 \mathrm{H}, \mathrm{H} 5, J=1.8 \mathrm{~Hz}), 6.9(\mathrm{~d}, 1 \mathrm{H}, \mathrm{H} 3, J=1.8 \mathrm{~Hz})$, $5.9-5.8\left(\mathrm{~m}, 1 \mathrm{H}, \mathrm{CH}_{2}=\mathrm{CH}-\mathrm{CH}_{2}\right), 5.1-5.0\left(\mathrm{~m}, 2 \mathrm{H}, \mathrm{CH}_{2}=\mathrm{CH}-\right.$ $\left.\mathrm{CH}_{2}\right), 3.9\left(\mathrm{~s}, 3 \mathrm{H}, \mathrm{OCH}_{3}\right), 3.3\left(\mathrm{~d}, 2 \mathrm{H}, \mathrm{CH}_{2}=\mathrm{CH}-\overline{\mathrm{CH}}_{2}, J=\right.$ $6.6 \mathrm{~Hz}) ;{ }^{13} \mathrm{C} \mathrm{NMR}\left(\delta ; \mathrm{CDCl}_{3} ; 75 \mathrm{MHz}\right): 149.7(\mathrm{C} 6), 144.7(\mathrm{C} 1)$, $135.8\left(\mathrm{CH}_{2}=\mathrm{CH}-\mathrm{CH}_{2}\right), 133.5(\mathrm{C} 2), 131.1(\mathrm{C} 4), 118.5$ (C5), 117.0 $\left(\underline{\mathrm{CH}}_{2}=\mathrm{CH}-\overline{\mathrm{CH}}_{2}\right), 114.9(\mathrm{C} 3), 56.6\left(\mathrm{OCH}_{3}\right), 39.3\left(\mathrm{CH}_{2}=\mathrm{CH}-\right.$ $\left.\underline{\mathrm{CH}}_{2}\right)$.

2-Methoxy-6-nitro-4-propylphenol $\left(2^{\prime}\right)$. Yellow oil; 62\% yield; IR $\left(\bar{v}_{\text {máx }}\right.$, in cm $\left.{ }^{-1}\right): 3250(\mathrm{O}-\mathrm{H}), 1591(\mathrm{C}=\mathrm{C}), 1541,1448\left(\mathrm{NO}_{2}\right)$, $1260(\mathrm{C}-\mathrm{O}-\mathrm{C}) ;{ }^{1} \mathrm{H} \mathrm{NMR}\left(\delta ; \mathrm{CDCl}_{3} ; 300 \mathrm{MHz}\right): 10.6$ (s, $1 \mathrm{H}, \mathrm{O}-$ $\underline{\mathrm{H}}), 7.4(\mathrm{~d}, 1 \mathrm{H}, \mathrm{H} 5, J=1.8 \mathrm{~Hz}), 6.9(\mathrm{~d}, 1 \mathrm{H}, \mathrm{H} 3, J=1.8 \mathrm{~Hz}), 3.9$ $\left(\mathrm{s}, 3 \mathrm{H}, \mathrm{OCH}_{3}\right), 2.5$ (t, $\left.2 \mathrm{H}, \mathrm{CH}_{3}-\mathrm{CH}_{2}-\underline{\mathrm{CH}}_{2}, J=7.2 \mathrm{~Hz}\right), 1.7-1.5$ (m, $\left.2 \mathrm{H}, \mathrm{CH}_{3}-\mathrm{CH}_{2}-\mathrm{CH}_{2}\right), 0.9\left(\mathrm{t}, 3 \mathrm{H}, \underline{\mathrm{C}}_{3}-\mathrm{CH}_{2}-\mathrm{CH}_{2}, J=\right.$ $7.2 \mathrm{~Hz}) ;{ }^{13} \mathrm{C} \mathrm{NMR}\left(\delta ; \mathrm{CDCl}_{3} ; 75 \mathrm{MHz}\right): 149.6$ (C6), $144.5(\mathrm{Cl})$, 133.7 (C4), 133.5 (C2), 118.6 (C5), 114.7 (C3), $56.6\left(\mathrm{OC}_{3}\right)$, $37.3\left(\mathrm{CH}_{3}-\mathrm{CH}_{2}-\underline{\mathrm{CH}_{2}}\right), 24.1\left(\mathrm{CH}_{3}-\underline{\mathrm{CH}_{2}}-\mathrm{CH}_{2}\right), 13.5\left(\underline{\mathrm{CH}_{3}-\mathrm{CH}_{2}-}\right.$ $\left.\mathrm{CH}_{2}\right)$.

Synthesis of Amino Derivatives $\mathbf{3}$ and $\mathbf{3}^{\prime}$. The nitrocompounds $2(11.87 \mathrm{mmol})$ or $\mathbf{2}^{\prime}(19.43 \mathrm{mmol})$ were solubilized in ethanol $(100 \mathrm{~mL})$ and to these solutions was added dihydrate stannous chloride (5 eq.). The mixture was kept under magnetic stirring at $70^{\circ} \mathrm{C}$ for 3 hours and the consumption of starting material was followed by TLC (chloroform/methanol, $9.5: 0.5, \mathrm{v} / \mathrm{v}$ ). The mixture was poured into ice water and aqueous $\mathrm{NaHCO} 3$ adjusted the $\mathrm{pH}$ to 7 . The mixture was then extracted with ethyl acetate $(10 \times 20 \mathrm{~mL})$; the organic layer was dried with anhydrous sodium sulfate and filtered, and the filtrate was concentrated under reduced pressure, which provided the pure products.

4-Allyl-2-amino-6-methoxyphenol (3). Yellow crystals; $61 \%$ yield; m.p.: $95-98^{\circ} \mathrm{C}$; IR ( $\bar{v}_{\text {máx }}$, in $\left.\mathrm{cm}^{-1}\right): 3371,3311\left(\mathrm{NH}_{2}\right)$, 3074 (O-H), 1606, 1456 (C=C), 1512 (N-H), 1230 (C-O-C); ${ }^{1} \mathrm{H} \operatorname{NMR}\left(\delta ; \mathrm{CDCl}_{3} ; 300 \mathrm{MHz}\right): 6.2(\mathrm{~d}, 1 \mathrm{H}, \mathrm{H} 5, J=1.8 \mathrm{~Hz})$, $6.1(\mathrm{~d}, 1 \mathrm{H}, \mathrm{H} 3, J=1.8 \mathrm{~Hz}), 6.0-5.8\left(\mathrm{~m}, 1 \mathrm{H}, \mathrm{CH}_{2}=\mathrm{CH}-\mathrm{CH}_{2}\right)$, 5.1-5.0 (m, $\left.2 \mathrm{H}, \mathrm{CH}_{2}=\mathrm{CH}-\mathrm{CH}_{2}\right), 3.8\left(\mathrm{~s}, 3 \mathrm{H}, \mathrm{OCH}_{\underline{3}}\right), 3,2(\mathrm{~d}, 2 \mathrm{H}$, $\left.\mathrm{CH}_{2}=\mathrm{CH}-\mathrm{CH}_{2}, J=6.9 \mathrm{~Hz}\right) ;{ }^{13} \mathrm{C} \mathrm{NMR}\left(\delta ; \mathrm{CDCl}_{3} ; 75 \mathrm{MHz}\right)$ : 146.6 (C6), $137.8\left(\mathrm{CH}_{2}=\mathrm{CH}-\mathrm{CH}_{2}\right), 133.8$ (C2), 131.6 (C4), $131.2(\mathrm{C} 1), 115.3\left(\mathrm{CH}_{2}=\mathrm{CH}-\mathrm{CH}_{2}\right), 109.4$ (C5), 101.9 (C3), 55.9 $\left(\mathrm{OCH}_{3}\right), 40.0\left(\mathrm{CH}_{2}=\mathrm{CH}-\underline{\mathrm{CH}}_{2}\right)$.

2-Amino-6-methoxy-4-propylphenol ( $\left.3^{\prime}\right)$. Yellow crystals; $46 \%$ yield; m.p.: $63-66^{\circ} \mathrm{C}$; IR ( $\bar{v}_{\text {máx }}$, in $\left.\mathrm{cm}^{-1}\right): 3370,3307\left(\mathrm{NH}_{2}\right)$, 3012 (O-H), 1513 (N-H), 1212 (C-O-C); ${ }^{1} \mathrm{H}$ NMR $\left(\delta ; \mathrm{CDCl}_{3}\right.$; $300 \mathrm{MHz}): 6.2(\mathrm{~d}, 1 \mathrm{H}, \mathrm{H} 5, J=1.8 \mathrm{~Hz}) ; 6.1(\mathrm{~d}, 1 \mathrm{H}, \mathrm{H} 3, J=$ $1.8 \mathrm{~Hz}), 3.8\left(\mathrm{~s}, 3 \mathrm{H}, \mathrm{OC}_{\underline{3}}\right), 2.4\left(\mathrm{t}, 2 \mathrm{H}, \mathrm{CH}_{3}-\mathrm{CH}_{2}-\mathrm{CH}_{2}, J=\right.$ 
7.2 Hz), 1.6-1.5 (m, $\left.2 \mathrm{H}, \mathrm{CH}_{3}-\mathrm{C}_{2}-\mathrm{CH}_{2}\right), 0.9$ (t, 3H, $\underline{\mathrm{C}}_{3}-$ $\left.\mathrm{CH}_{2}-\mathrm{CH}_{2}, J=7.5 \mathrm{~Hz}\right) ;{ }^{13} \mathrm{C}$ NMR $\left(\delta ; \mathrm{CDCl}_{3} ; 75 \mathrm{MHz}\right): 146.4$ (C6), 134.3 (C2), 133.7 (C4), 130.8 (C1), 109.3 (C5), 101.7 (C3), 55,92 $\left(\mathrm{OCH}_{3}\right), 37,99\left(\mathrm{CH}_{3}-\mathrm{CH}_{2}-\mathrm{CH}_{2}\right), 24,74\left(\mathrm{CH}_{3}-\right.$ $\left.\mathrm{CH}_{2}-\mathrm{CH}_{2}\right), 13,82\left(\mathrm{CH}_{3}-\mathrm{CH}_{2}-\mathrm{CH}_{2}\right)$.

Synthesis of Amides $\mathbf{4 a}-\mathbf{4} \boldsymbol{d}$ and $\mathbf{4} \boldsymbol{a}^{\prime}-\mathbf{4} \boldsymbol{d}^{\prime}$. To a solution of 3 or $\mathbf{3}^{\prime}$ (1 eq.) in diethyl ether $(5 \mathrm{~mL})$ was added $5 \mathrm{~mL}$ of aqueous $\mathrm{NaHCO}_{3}$ (1 eq.) and this mixture was stirred at $0^{\circ} \mathrm{C}$ for $5 \mathrm{~min}$. Then, a solution of the corresponding acyl chloride (1 eq.) in $5 \mathrm{~mL}$ of ethyl ether was added dropwise over 30 minutes to the reaction mixture. Then the mixture was stirred at room temperature for 2 hours and the progress of the reaction was monitored by TLC (hexane/ethyl acetate, $7: 3, \mathrm{v} / \mathrm{v})$. The compound $4 \mathrm{a}$ was isolated by filtration and the others were isolated by extraction with dichloromethane, after evaporation of the diethyl ether. When $O$-acylation byproducts were detected, the crude product was treated with $\mathrm{NaHCO}_{3}$ (1 eq.) in aqueous methanol for 24 hours at room temperature. After deacylation (checked by TLC, hexane/ethyl acetate, $7: 3, \mathrm{v} / \mathrm{v}$ ), water was removed with anhydrous sodium sulfate, the mixture was filtered, and the solvent was removed under reduced pressure.

N-(5-Allyl-2-hydroxy-3-methoxyphenyl)benzamide (4a). Light brown semi-solid; $40 \%$ yield; ${ }^{1} \mathrm{H}$ NMR $\left(\delta ; \mathrm{CDCl}_{3}\right.$; $300 \mathrm{MHz}): 8.3(\mathrm{~s} ; 1 \mathrm{H} ; \mathrm{N}-\underline{\mathrm{H}}), 7.9-7.8\left(\mathrm{~m} ; 2 \mathrm{H} ; \mathrm{H}^{\prime}\right), 7.7$ (d; $1 \mathrm{H}$; $\mathrm{H} 6 ; J=1.8 \mathrm{~Hz}) ; 7.5-7.4\left(\mathrm{~m} ; 3 \mathrm{H}, \mathrm{H}^{\prime}\right.$ e $\left.\mathrm{H}^{\prime}\right), 6.5(\mathrm{~d} ; 1 \mathrm{H} ; \mathrm{H} 4$; $J=1.8 \mathrm{~Hz}), 6.0-5.8\left(\mathrm{~m} ; 1 \mathrm{H} ; \mathrm{CH}_{2}=\mathrm{CH}-\mathrm{CH}_{2}-\right), 5.1-5.0(\mathrm{~m} ; 2 \mathrm{H}$; $\left.\mathrm{CH}_{2}=\mathrm{CH}-\mathrm{CH}_{2}\right), 3.8\left(\mathrm{~s} ; 3 \mathrm{H} ; \mathrm{OCH}_{3}\right), 3.3\left(\mathrm{~d} ; 2 \mathrm{H} ; \mathrm{CH}_{2}=\mathrm{CH}-\right.$ $\left.\mathrm{C}_{2} ; J=6.6 \mathrm{~Hz}\right) .{ }^{13} \mathrm{CNMR}\left(\delta ; \mathrm{CDCl}_{3} ; 75 \mathrm{MHz}\right): 165.6(\underline{\mathrm{C}}=\mathrm{O})$, $146.8(\mathrm{C} 3), 137.5\left(\mathrm{CH}_{2}=\mathrm{CH}-\mathrm{CH}_{2}\right), 134.6\left(\mathrm{Cl}^{\prime}\right), 133.6(\mathrm{C} 2), 131.9$ (C5), $131.8\left(\mathrm{C}^{\prime}\right), 128.7\left(\mathrm{C}^{\prime}\right), 127.1\left(\mathrm{C}^{\prime}\right), 125.8(\mathrm{Cl}), 115.7$ $\left(\mathrm{CH}_{2}=\mathrm{CH}-\mathrm{CH}_{2}\right), 113.3(\mathrm{C} 6), 107.2(\mathrm{C} 4), 56.1\left(\mathrm{OCH}_{3}\right), 40.2$ $\left(\overline{\mathrm{C}} \mathrm{H}_{2}=\mathrm{CH}-\mathrm{CH}_{2}\right)$.

N-(5-Allyl-2-hydroxy-3-methoxyphenyl)-4-chlorobenzamide (4b). Pink crystals; $64 \%$ yield; ${ }^{1} \mathrm{H}$ NMR $\left(\delta ; \mathrm{CDCl}_{3}\right.$; $300 \mathrm{MHz}$ ): 8.3 (s; $1 \mathrm{H} ; \mathrm{N}-\underline{\mathrm{H}}$ ), 7.8 (d; $2 \mathrm{H} ; \mathrm{H}^{\prime} ; J=8.1 \mathrm{~Hz}$ ); $7.7(\mathrm{~s} ; 1 \mathrm{H} ; \mathrm{H} 6) ; 7.4\left(\mathrm{~d} ; 2 \mathrm{H} ; \mathrm{H}^{\prime} ; J=8.1 \mathrm{~Hz}\right), 6.5(\mathrm{~s} ; 1 \mathrm{H} ; \mathrm{H} 4)$, 6.0-5.8 (m;1H; $\left.\mathrm{CH}_{2}=\mathrm{C} \underline{\mathrm{H}}-\mathrm{CH}_{2}-\right), 5.1-5.0\left(\mathrm{~m} ; 2 \mathrm{H} ; \mathrm{CH}_{2}=\mathrm{CH}-\right.$ $\left.\mathrm{CH}_{2}\right), 3.8\left(\mathrm{~s} ; 3 \mathrm{H} ; \mathrm{OC}_{\underline{3}}\right), 3.3\left(\mathrm{~d} ; 2 \mathrm{H} ; \mathrm{CH}_{2}=\mathrm{CH}-\overline{\mathrm{CH}}_{2} ; J=\right.$ $6.6 \mathrm{~Hz}) .{ }^{13} \mathrm{C} \mathrm{NMR}\left(\delta ; \mathrm{CDCl}_{3} ; 75 \mathrm{MHz}\right): 164.4(\underline{\mathrm{C}}=\mathrm{O}), 146.6$ (C3), $138.1\left(\mathrm{C}^{\prime}\right), 137.4\left(\mathrm{CH}_{2}=\mathrm{CH}-\mathrm{CH}_{2}\right), 133.5(\mathrm{C} 2), 133.1$ $\left(\mathrm{Cl}^{\prime}\right), 132.0$ (C5), $128.9\left(\mathrm{C} 2^{\prime}\right), 128.5\left(\mathrm{C}^{\prime}\right), 125.5(\mathrm{Cl}), 115.8$ $\left(\mathrm{CH}_{2}=\mathrm{CH}-\mathrm{CH}_{2}\right), 113.2(\mathrm{C} 6), 107.3(\mathrm{C} 4), 56.1\left(\mathrm{OCH}_{3}\right), 40.2$ $\left(\overline{\mathrm{C}} \mathrm{H}_{2}=\mathrm{CH}-\mathrm{CH}_{2}\right)$.

N-(5-Allyl-2-hydroxy-3-methoxyphenyl)-4-methoxybenzamide (4c). Orange crystals; $67 \%$ yield; ${ }^{1} \mathrm{H}$ NMR $\left(\delta ; \mathrm{CDCl}_{3}\right.$; $300 \mathrm{MHz}): 7.9-7.8\left(\mathrm{~m} ; 2 \mathrm{H} ; \mathrm{H} 2^{\prime}\right), 7.6(\mathrm{~d} ; 1 \mathrm{H} ; \mathrm{H} 6 ; J=1.8 \mathrm{~Hz})$, 7.0-6.9 (m; 2H; H3'), $6.5(\mathrm{~d} ; 1 \mathrm{H} ; \mathrm{H} 4 ; J=1.8 \mathrm{~Hz}), 6.0-5.8$ (m; $\left.1 \mathrm{H} ; \mathrm{CH}_{2}=\mathrm{C} \underline{\mathrm{H}}-\mathrm{CH}_{2}-\right)$, 5.1-5.0 (m; $2 \mathrm{H} ; \mathrm{C}_{2}=\mathrm{CH}-\mathrm{CH}_{2}$ ), 3.8 (s; $\left.3 \mathrm{H} ; \mathrm{C}^{-}-\mathrm{OC}_{3}\right), 3.8$ (s; $\left.3 \mathrm{H} ; \mathrm{C}^{\prime}-\mathrm{OC}_{3}\right), 3.3$ (d; $2 \mathrm{H}$; $\left.\mathrm{CH}_{2}=\mathrm{CH}-\underline{\mathrm{CH}}_{2} ; J=6.6 \mathrm{~Hz}\right) .{ }^{13} \mathrm{C} \mathrm{NMR}\left(\delta ; \mathrm{CDCl}_{3} ; 75 \mathrm{MHz}\right)$ : $165.1(\underline{\mathrm{C}}=\mathrm{O}), 162.5\left(\mathrm{C}^{\prime}\right), 146.9(\mathrm{C} 3), 137.5\left(\mathrm{CH}_{2}=\mathrm{CH}-\mathrm{CH}_{2}\right)$, 133.7 (C2), 131.9 (C5), $129.0\left(\mathrm{C}^{\prime}\right), 126.8(\mathrm{Cl}), 125.9\left(\mathrm{Cl}^{\prime}\right)$,
$115.7\left(\mathrm{CH}_{2}=\mathrm{CH}-\mathrm{CH}_{2}\right), 113.9\left(\mathrm{C}^{\prime}\right), 113.3(\mathrm{C} 6), 107.1(\mathrm{C} 4), 56.1$ $\left(\mathrm{C} 3-\underline{\mathrm{O}}_{3}\right), 55.4\left(\mathrm{C} 4^{\prime}-\mathrm{OCH}_{3}\right), 40.2\left(\mathrm{CH}_{2}=\mathrm{CH}-\underline{\mathrm{CH}}_{2}\right)$.

N-(5-Allyl-2-hydroxy-3-methoxyphenyl)-4-nitrobenzamide $(4 d)$. Yellow crystals; $86 \%$ yield; ${ }^{1} \mathrm{H}$ NMR $\left(\delta ; \mathrm{CDCl}_{3}\right.$; $300 \mathrm{MHz}): 8.3(\mathrm{~s} ; 1 \mathrm{H} ; \mathrm{N}-\underline{\mathrm{H}}), 8.3-8.2\left(\mathrm{~m} ; 2 \mathrm{H} ; \mathrm{H}^{\prime}\right), 8.1-8.0$ (m; $\left.2 \mathrm{H} ; \mathrm{H}^{\prime}\right), 7.7(\mathrm{~s} ; 1 \mathrm{H}, \overline{\mathrm{O}}-\underline{\mathrm{H}}), 6.5(\mathrm{~d} ; 1 \mathrm{H} ; \mathrm{H} 6 ; J=1.8 \mathrm{~Hz})$, $6.0(\mathrm{~d} ; 1 \mathrm{H} ; \mathrm{H} 4 ; J=1.8 \mathrm{~Hz}), 6.0-5.8\left(\mathrm{~m} ; 1 \mathrm{H} ; \mathrm{CH}_{2}=\mathrm{CH}-\mathrm{CH}_{2}-\right)$, 5.1-5.0 (m;2H; $\left.\mathrm{CH}_{2}=\mathrm{CH}-\mathrm{CH}_{2}\right), 3.8\left(\mathrm{~s} ; 3 \mathrm{H} ; \mathrm{OCH}_{3}\right), 3.3$ $\left(\mathrm{d} ; 2 \mathrm{H} ; \mathrm{CH}_{2}=\mathrm{CH}-\mathrm{CH}_{2} ; J=6.9 \mathrm{~Hz}\right) .{ }^{13} \mathrm{C}$ NMR $\left(\delta ; \mathrm{CDCl}_{3}\right.$; $75 \mathrm{MHz}): 163.2(\mathrm{C}=\mathrm{O}), 149.6\left(\mathrm{C}^{\prime}\right), 146.4(\mathrm{C} 3), 140.3\left(\mathrm{Cl}^{\prime}\right)$, $137.3\left(\mathrm{CH}_{2}=\mathrm{C} H-\mathrm{CH}_{2}\right), 133.4(\mathrm{C} 2), 132.2(\mathrm{C} 5), 128.2\left(\mathrm{C}^{\prime}\right)$, $125.1(\mathrm{Cl}), 123.9\left(\mathrm{C}^{\prime}\right), 115.9\left(\mathrm{CH}_{2}=\mathrm{CH}-\mathrm{CH}_{2}\right), 113.2(\mathrm{C} 6)$, 107.6 (C4), $56.1\left(\mathrm{OCH}_{3}\right), 40.1\left(\mathrm{CH}_{2}=\mathrm{CH}-\underline{C H}_{2}\right)$.

$N$-(2-Hydroxy-3-methoxy-5-propylphenyl)benzamide $\left(\mathbf{4} \boldsymbol{a}^{\prime}\right)$. Orange oil; $39 \%$ yield; ${ }^{1} \mathrm{H}$ NMR $\left(\delta ; \mathrm{CDCl}_{3} ; 300 \mathrm{MHz}\right): 8.3$ (s; $1 \mathrm{H} ; \mathrm{N}-\underline{\mathrm{H}}), 7.9-7,8\left(\mathrm{~m} ; 2 \mathrm{H} ; \mathrm{H}^{\prime}\right), 7.7(\mathrm{~d} ; 1 \mathrm{H} ; \mathrm{H} 6 ; J=1.5 \mathrm{~Hz})$, 7.5-7.4 (m; $2 \mathrm{H} ; \mathrm{H}^{\prime}{ }^{\prime}$ e $\left.\mathrm{H}^{\prime}{ }^{\prime}\right), 6.5$ (d; $\left.1 \mathrm{H} ; \mathrm{H} 4 ; J=1.8 \mathrm{~Hz}\right), 6.1$ (s; $1 \mathrm{H} ; \mathrm{O}-\underline{\mathrm{H}}), 3.8\left(\mathrm{~s} ; 3 \mathrm{H} ; \mathrm{OC}_{3}\right), 2.5\left(\mathrm{t} ; 2 \mathrm{H} ; \mathrm{CH}_{3}-\mathrm{CH}_{2}-\mathrm{CH}_{2}-;\right.$ $=7.5 \mathrm{~Hz}), 1.7-1.5\left(\mathrm{~m} ; 2 \mathrm{H} ; \overline{\mathrm{CH}}_{3}-\mathrm{CH}_{2}-\mathrm{CH}_{2}-\right), 0.9$ (t; $3 \mathrm{H} ; \underline{\mathrm{CH}}_{3}-$ $\left.\mathrm{CH}_{2}-\mathrm{CH}_{2}-; J=7.2 \mathrm{~Hz}\right) \cdot{ }^{13} \mathrm{C}$ NMR $\left(\delta ; \mathrm{CDCl}_{3} ; 75 \mathrm{MHz}\right): 165.5$ $(\underline{\mathrm{C}}=\mathrm{O}), 146.5(\mathrm{C} 3), 134.7(\mathrm{C} 2), 133.6\left(\mathrm{Cl}^{\prime}\right), 133.2(\mathrm{C} 5), 131.8$ $\left(\overline{\mathrm{C}} 4^{\prime}\right), 128.7\left(\mathrm{C}^{\prime}\right), 127.1\left(\mathrm{C}^{\prime}\right), 125.6(\mathrm{C} 1), 113.0(\mathrm{C} 6), 107.2(\mathrm{C} 4)$, $56.1\left(\mathrm{OCH}_{3}\right), 38.1\left(\mathrm{CH}_{3}-\mathrm{CH}_{2}-\mathrm{CH}_{2}-\right), 24.8\left(\mathrm{CH}_{3}-\mathrm{CH}_{2}-\mathrm{CH}_{2}-\right)$, $13.8\left(\mathrm{C}_{3}-\mathrm{CH}_{2}-\mathrm{CH}_{2}-\right)$.

$\mathrm{N}$-(2-Hydroxy-3-methoxy-5-propylphenyl)-4-chlorobenzamide $\left(\mathbf{4} \boldsymbol{b}^{\prime}\right)$. White crystals; $37 \%$ yield; ${ }^{1} \mathrm{H}$ NMR $\left(\delta ; \mathrm{CDCl}_{3}\right.$; $300 \mathrm{MHz}): 8.2(\mathrm{~s} ; 1 \mathrm{H} ; \mathrm{N}-\underline{\mathrm{H}}), 7.9-7.8\left(\mathrm{~m} ; 2 \mathrm{H} ; \mathrm{H}^{\prime}\right), 7.7$ (d; $1 \mathrm{H}$; $\mathrm{H} 6 ; J=1.8 \mathrm{~Hz}), 7.5-7.4\left(\mathrm{~m} ; 2 \mathrm{H} ; \mathrm{H}^{\prime}\right), 6.5(\mathrm{~d} ; 1 \mathrm{H} ; \mathrm{H} 4 ; J=$ $1.8 \mathrm{~Hz}), 5.9(\mathrm{~s} ; 1 \mathrm{H} ; \mathrm{O}-\underline{\mathrm{H}}), 3.8\left(\mathrm{~s} ; 3 \mathrm{H} ; \mathrm{OC}_{3}\right), 2.5\left(\mathrm{t} ; 2 \mathrm{H} ; \mathrm{CH}_{3}-\right.$ $\left.\mathrm{CH}_{2}-\mathrm{C}_{2}-; J=7.5 \mathrm{~Hz}\right), 1.7-1.5\left(\mathrm{~m} ; 2 \mathrm{H} ; \mathrm{CH}_{3}-\mathrm{CH}_{2}-\mathrm{CH}_{2}-\right), 0.9$ $\left(\mathrm{t} ; 3 \mathrm{H} ; \mathrm{CH}_{3}-\mathrm{CH}_{2}-\mathrm{CH}_{2}-; J=7.2 \mathrm{~Hz}\right) .{ }^{13} \mathrm{C} \mathrm{NMR}\left(\delta ; \mathrm{CDCl}_{3}\right.$; $75 \mathrm{MHz}): 1 \overline{6} 4.3(\underline{\mathrm{C}}=\mathrm{O}), 146.3(\mathrm{C} 3), 138.1\left(\mathrm{C}^{\prime}\right), 134.8$ (C2), $133.2(\mathrm{C} 1), 133.0(\mathrm{C} 5), 129.0\left(\mathrm{C}^{\prime}\right), 128.5\left(\mathrm{C}^{\prime}\right), 125.4(\mathrm{C} 1)$, 113.0 (C6), $107.2(\mathrm{C} 4), 56.1\left(\mathrm{OCH}_{3}\right), 38.1\left(\mathrm{CH}_{3}-\mathrm{CH}_{2}-\mathrm{CH}_{2}-\right)$, $24.8\left(\mathrm{CH}_{3}-\mathrm{CH}_{2}-\mathrm{CH}_{2}-\right), 13.7\left(\underline{\mathrm{CH}}_{3}-\mathrm{CH}_{2}-\mathrm{CH}_{2}-\right)$.

N-(2-Hydroxy-3-methoxy-5-propylphenyl)-4-methoxybenzamide $\left(\mathbf{4} \boldsymbol{c}^{\prime}\right)$. Brown crystals; $95 \%$ yield; ${ }^{1} \mathrm{H}$ NMR $\left(\delta ; \mathrm{CDCl}_{3}\right.$; $300 \mathrm{MHz}): 8.2(\mathrm{~s} ; 1 \mathrm{H} ; \mathrm{N}-\mathrm{H}), 7.9-7.8\left(\mathrm{~m} ; 2 \mathrm{H} ; \mathrm{H}^{\prime}\right), 7.6(\mathrm{~d} ; 1 \mathrm{H}$; $\mathrm{H} 6 ; J=1.5 \mathrm{~Hz}), 7.0-6.9\left(\mathrm{~m} ; 2 \mathrm{H} ; \mathrm{H}^{\prime}\right), 6.5(\mathrm{~d} ; 1 \mathrm{H} ; \mathrm{H} 4 ; J=$ $1.8 \mathrm{~Hz}), 3.9\left(\mathrm{~s} ; 3 \mathrm{H} ; \mathrm{C} 3-\mathrm{OCH}_{3}\right), 3.8\left(\mathrm{~s} ; 3 \mathrm{H} ; \mathrm{C4}^{\prime}-\mathrm{OCH}_{3}\right), 2.5$ (t; $\left.2 \mathrm{H} ; \mathrm{CH}_{3}-\mathrm{CH}_{2}-\mathrm{CH}_{2}-; J=7.5 \mathrm{~Hz}\right), 1.6-1.5\left(\mathrm{~m} ; 2 \mathrm{H} ; \mathrm{CH}_{3}-\right.$ $\left.\mathrm{CH}_{2}-\mathrm{CH}_{2}-\right), 0.9\left(\mathrm{t} ; 3 \mathrm{H} ; \mathrm{CH}_{3}-\mathrm{CH}_{2}-\mathrm{CH}_{2} ; \quad J=7.2 \mathrm{~Hz}\right) .{ }^{13} \mathrm{C}$ NMR $\left(\delta ; \mathrm{CDCl}_{3} ; 75 \mathrm{MHz}\right): 165.1(\underline{\mathrm{C}}=\mathrm{O}), 162.5\left(\mathrm{C}^{\prime}\right), 146.8$ (C3), 134.6 (C2), 133.4 (C5), $129.0\left(\mathrm{C}^{\prime}\right), 126.9$ (C1), 125.8 $\left(\mathrm{Cl}^{\prime}\right), 113.9\left(\mathrm{C3}^{\prime}\right), 113.1(\mathrm{C} 6), 107.1(\mathrm{C} 4), 56.1\left(\mathrm{C} 3-\mathrm{OCH}_{3}\right), 55.4$ $\left(\mathrm{C}^{\prime}-\mathrm{OCH}_{3}\right), 38.1\left(\mathrm{CH}_{3}-\mathrm{CH}_{2}-\mathrm{CH}_{2}-\right), 24.7\left(\mathrm{CH}_{3}-\underline{\mathrm{CH}}_{2}-\mathrm{CH}_{2}-\right)$, $13.7\left(\mathrm{CH}_{3}-\mathrm{CH}_{2}-\mathrm{CH}_{2}-\right)$.

N-(2-Hydroxy-3-methoxy-5-propylphenyl)-4-nitrobenzamide $\left(4 \boldsymbol{d}^{\prime}\right)$. Orange crystals; $41 \%$ yield; ${ }^{1} \mathrm{H}$ NMR $\left(\delta ; \mathrm{CDCl}_{3}\right.$; $300 \mathrm{MHz}): 8.4-8.3\left(\mathrm{~m} ; 2 \mathrm{H} ; \mathrm{H}^{\prime}\right), 8.1-8.0\left(\mathrm{~m} ; 2 \mathrm{H} ; \mathrm{H}^{\prime}\right)$ ), 7.8 (s; 1H; H6), 6.5 (d; 1H; H4; $J=1.8 \mathrm{~Hz}), 5.8(\mathrm{~s} ; 1 \mathrm{H} ; \mathrm{O}-\underline{\mathrm{H}}), 3.9$ (s; $3 \mathrm{H} ; \mathrm{OC}_{3}$ ), $2.5\left(\mathrm{t} ; 2 \mathrm{H} ; \mathrm{CH}_{3}-\mathrm{CH}_{2}-\mathrm{C}_{2}-; J=7.2 \mathrm{~Hz}\right), 1.7-1.5$ 
(m; $2 \mathrm{H} ; \mathrm{CH}_{3}-\mathrm{CH}_{2}-\mathrm{CH}_{2}-$ ), 0.9 (t; $3 \mathrm{H} ; \mathrm{C}_{3}-\mathrm{CH}_{2}-\mathrm{CH}_{2}-; \quad J=$ $7.2 \mathrm{~Hz}) .{ }^{13} \mathrm{C} \operatorname{NMR}\left(\delta ; \mathrm{CDCl}_{3} ; 75 \mathrm{MHz}\right): 163.1(\underline{\mathrm{C}}=\mathrm{O}), 149.7$ (C4'), 146.1 (C3), $140.5\left(\mathrm{Cl}^{\prime}\right), 134.9$ (C2), 132.9 (C5), 128.2 (C2'), $124.9(\mathrm{C}), 124.0\left(\mathrm{C}^{\prime}\right), 112.9$ (C6), 107.4 (C4), 56.1 $\left(\mathrm{OCH}_{3}\right), 38.1\left(\mathrm{CH}_{3}-\mathrm{CH}_{2}-\mathrm{CH}_{2}-\right), 24.8\left(\mathrm{CH}_{3}-\mathrm{CH}_{2}-\mathrm{CH}_{2}-\right), 13.7$ $\left(\mathrm{CH}_{3}-\mathrm{CH}_{2}-\mathrm{CH}_{2}-\right)$.

Synthesis of Benzoxazoles $\mathbf{5 a}-\mathbf{5} \boldsymbol{d}$ and $\mathbf{5} \boldsymbol{a}^{\prime}-\mathbf{5} \boldsymbol{d}^{\prime}$. To a solution of the corresponding amides $\left(\mathbf{4 a}-\mathbf{4 d}, \mathbf{4} \mathbf{a}^{\prime}-\mathbf{4} \mathbf{d}^{\prime}, 1\right.$ eq. $)$ in toluene $(25 \mathrm{~mL}$ ) was added $p$-toluenesulfonic acid (3 eq.) and the mixture was stirred under reflux. The evolution of the reaction was monitored by TLC (hexane/ethyl acetate, $7: 3, \mathrm{v} / \mathrm{v}$ ). After the end of reaction, toluene was removed under reduced pressure and the crude product obtained was purified by filtration over silica gel with dichloromethane (compounds $4 \mathrm{a}$, $4 \mathrm{~d}, 4 \mathrm{a}^{\prime}, 4 \mathrm{~b}^{\prime}$, and $4 \mathrm{~d}^{\prime}$ ) or by column chromatography (compounds $4 \mathrm{~b}, 4 \mathrm{c}, 4 \mathrm{c}^{\prime}$, hexane/ethyl acetate, $\left.9: 1, \mathrm{v} / \mathrm{v}\right)$.

5-Allyl-7-methoxy-2-phenylbenzo[d]oxazol (5a). Yellow semisolid; $36 \%$ yield; Rf 0.8 ; IR $\left(\bar{v}_{\text {máx }}\right.$, in $\left.\mathrm{cm}^{-1}\right): 1626(\mathrm{C}=\mathrm{N}), 1603$, 1585, 1449 (C=C), 1321, 1202 (C-N), 1132 (C-O-C); ${ }^{1} \mathrm{H}$ NMR $\left(\delta ; \mathrm{CDCl}_{3} ; 300 \mathrm{MHz}\right): 8.2-8.2\left(\mathrm{~m}, 2 \mathrm{H}, \mathrm{H}^{\prime}\right), 7.5-7.5(\mathrm{~m}, 2 \mathrm{H}$, $\left.\mathrm{H}^{\prime}\right), 7.5\left(\mathrm{~m}, 1 \mathrm{H}, \mathrm{H} 4^{\prime}\right), 7.3-7.15(\mathrm{~m}, 1 \mathrm{H}, \mathrm{H} 4), 6.7$ (d, 1H, H6; $J=1.5 \mathrm{~Hz}), 6.0-5.9\left(\mathrm{~m}, 1 \mathrm{H}, \mathrm{CH}_{2}=\mathrm{C} \underline{\mathrm{H}}-\mathrm{CH}_{2}\right), 5.1-5.0(\mathrm{~m}, 2 \mathrm{H}$, $\left.\mathrm{CH}_{2}=\mathrm{CH}-\mathrm{CH}_{2}\right), 4.0\left(\mathrm{~s}, 3 \mathrm{H}, \mathrm{OC}_{3}\right), 3.2\left(\mathrm{~d}, 2 \mathrm{H}, \mathrm{CH}_{2}=\mathrm{CH}-\right.$ $\left.\underline{\mathrm{C}}_{2} ; J=6.6 \mathrm{~Hz}\right) ;{ }^{13} \mathrm{C} \mathrm{NMR}\left(\delta ; \mathrm{CDCl}_{3} ; 75 \mathrm{MHz}\right): 144.3(\mathrm{C} 2)$, 143.9 (C7), 137.5 (C3a), $137.3\left(\mathrm{CH}_{2}=\mathrm{CH}-\mathrm{CH}_{2}\right), 131.4\left(\mathrm{C}^{\prime}\right)$, $128.8\left(\mathrm{C}^{\prime}\right), 127.6\left(\mathrm{C}^{\prime}\right), 127.1(\mathrm{C} 5), 125.7(\mathrm{C} 7 \mathrm{a}), 125.6\left(\mathrm{Cl}^{\prime}\right)$, $116.0\left(\mathrm{CH}_{2}=\mathrm{CH}-\mathrm{CH}_{2}\right), 112.0(\mathrm{C} 6), 108.8(\mathrm{C} 4), 56.3\left(\mathrm{OCH}_{3}\right)$, $40.5\left(\overline{\mathrm{CH}}_{2}=\mathrm{CH}-\mathrm{CH}_{2}\right)$. HRMS-ESI: $m / z$ calcd for $\mathrm{C}_{17} \mathrm{H}_{15} \mathrm{O}_{2} \mathrm{~N}$ $(\mathrm{M}+\mathrm{H})^{+}: 266.1176$; found: 266.1184 .

5-Allyl-2-(4-chlorophenyl)-7-methoxy-benzo[d]oxazol (5b). Beige crystals; $23 \%$ yield; m.p.: $103.8-105.5^{\circ} \mathrm{C}$; Rf 0.83; IR $\left(\bar{v}_{\text {máx }}\right.$, in $\left.\mathrm{cm}^{-1}\right): 1624(\mathrm{C}=\mathrm{N}), 1596,1578,1495(\mathrm{C}=\mathrm{C}), 1317,1199$ $(\mathrm{C}-\mathrm{N}), 1131$ (C-O-C); ${ }^{1} \mathrm{H}$ NMR $\left(\delta ; \mathrm{CDCl}_{3} ; 300 \mathrm{MHz}\right): 8.2-8.1$ (m, 2H, H2 $\left.{ }^{\prime}\right)$, 7.4-7.4 (m, 2H, H3' $)$, 7.2-6.09 (m, 1H, H4), 6.7 (d, $1 \mathrm{H}, \mathrm{H} 6 ; J=1.2 \mathrm{~Hz}), 6.0-5.9\left(\mathrm{~m}, 1 \mathrm{H}, \mathrm{CH}_{2}=\mathrm{CH}-\mathrm{CH}_{2}\right)$, 5.1-5.0 (m, $\left.2 \mathrm{H}, \mathrm{CH}_{2}=\mathrm{CH}-\mathrm{CH}_{2}\right), 4.0\left(\mathrm{~s}, 3 \mathrm{H}, \mathrm{OC}_{-}\right), 3.4$ $\left(\mathrm{d}, 2 \mathrm{H}, \mathrm{CH}_{2}=\mathrm{CH}-\underline{\mathrm{CH}}_{2} ; J=6.6 \mathrm{~Hz}\right) ;{ }^{13} \mathrm{C}$ NMR $\left(\delta ; \mathrm{CDCl}_{3}\right.$; $75 \mathrm{MHz}$ ): 162.0 (C2), 144.3 (C7), 143.8 (C3a), 138.5 (C5), 137.7 $\left(\mathrm{C}^{\prime}\right), 137.6(\mathrm{C} 7 \mathrm{a}), 137.2\left(\mathrm{CH}_{2}=\mathrm{CH}-\mathrm{CH}_{2}\right), 129.1\left(\mathrm{C}^{\prime}\right), 128.8$ $\left(\mathrm{C}^{\prime}\right), 125.6\left(\mathrm{Cl}^{\prime}\right), 116.1\left(\mathrm{CH}_{2}=\overline{\mathrm{C}} \mathrm{H}-\mathrm{CH}_{2}\right), 112.0(\mathrm{C} 6), 108.9$ (C4), $56.3\left(\mathrm{OCH}_{3}\right), 40.5\left(\mathrm{CH}_{2}=\mathrm{CH}-\mathrm{CH}_{2}\right)$. HRMS-ESI: $\mathrm{m} / z$ calcd for $\mathrm{C}_{17} \mathrm{H}_{14} \mathrm{O}_{2} \mathrm{NCl}(\mathrm{M}+\mathrm{H})^{+}: 300.0785$; found: 300.0794 .

5-Allyl-7-methoxy-2-(4-methoxyphenyl)benzo[d]oxazol (5c). Yellow crystals; $20 \%$ yield; m.p.: $81.3-84.1^{\circ} \mathrm{C}$; Rf 0.66; IR ( $\bar{v}_{\text {máx }}$, in $\left.\mathrm{cm}^{-1}\right): 1622(\mathrm{C}=\mathrm{N}), 1603,1582,1492(\mathrm{C}=\mathrm{C}), 1322,1248(\mathrm{C}-$ $\mathrm{N}), 1131(\mathrm{C}-\mathrm{O}-\mathrm{C}) ;{ }^{1} \mathrm{H}$ NMR $\left(\delta ; \mathrm{CDCl}_{3} ; 300 \mathrm{MHz}\right): 8.2-8.1$ $\left(\mathrm{m}, 2 \mathrm{H}, \mathrm{H} 2^{\prime}\right), 7.2-7.0(\mathrm{~m}, 1 \mathrm{H}, \mathrm{H} 4), 7.0-6.9\left(\mathrm{~m}, 2 \mathrm{H}, \mathrm{H}^{\prime}\right)$, $6.6(\mathrm{~d}, 1 \mathrm{H}, \mathrm{H} 6 ; J=1.2 \mathrm{~Hz}), 6.0-5.9\left(\mathrm{~m}, 1 \mathrm{H}, \mathrm{CH}_{2}=\mathrm{CH}-\mathrm{CH}_{2}\right)$, 5.1-5.0 (m, $\left.2 \mathrm{H}, \underline{\mathrm{CH}}_{2}=\mathrm{CH}-\mathrm{CH}_{2}\right), 4.0\left(\mathrm{~s}, 3 \mathrm{H}, \mathrm{C} 7-\mathrm{OC}_{3}\right), 3.8$ (s, $\left.3 \mathrm{H}, \mathrm{C} 4-\mathrm{OC}_{3}\right), 3.4$ (d, $2 \mathrm{H}, \mathrm{CH}_{2}=\mathrm{CH}-\underline{\mathrm{C}}_{2} ; J=6.6 \mathrm{~Hz}$ ); ${ }^{13} \mathrm{C} \mathrm{NMR}\left(\delta ; \mathrm{CDCl}_{3} ; 75 \mathrm{MHz}\right): 163.2(\mathrm{C} 2), 162.2\left(\mathrm{C}^{\prime}\right)$, 144.2 (C7), 144.0 (C3a), $137.4\left(\mathrm{CH}_{2}=\mathrm{C} H-\mathrm{CH}_{2}\right), 137.3$ (C5), $129.3\left(\mathrm{C}^{\prime}\right), 119.6\left(\mathrm{Cl}^{\prime}\right), 115.9\left(\mathrm{CH}_{2}=\overline{\mathrm{CH}}-\mathrm{CH}_{2}\right), 114.2\left(\mathrm{C}^{\prime}\right)$,
111.7 (C6), $108.4(\mathrm{C} 4), 56.3\left(\mathrm{C} 7-\mathrm{OCH}_{3}\right), 55.4\left(\mathrm{C}^{\prime}-\mathrm{OCH}_{3}\right)$, $40.5\left(\mathrm{CH}_{2}=\mathrm{CH}-\mathrm{CH}_{2}\right)$. HRMS-ESI: $\bar{m} / z$ calcd for $\mathrm{C}_{18} \mathrm{H}_{17} \mathrm{O}_{3} \mathrm{~N}$ $(\mathrm{M}+\mathrm{H})^{+}: 296.1281$; found: 296.1291 .

5-Allyl-7-methoxy-2-(4-nitrophenyl)benzo[d]oxazol (5d). Yellow crystals; $81 \%$ yield; m.p.: $161.2-164^{\circ} \mathrm{C}$; Rf 0.76; IR ( $\bar{v}_{\text {máx }}$, in $\left.\mathrm{cm}^{-1}\right): 1618(\mathrm{C}=\mathrm{N}), 1604,1459(\mathrm{C}=\mathrm{C}), 1518,1342\left(\mathrm{NO}_{2}\right), 1319$, $1202(\mathrm{C}-\mathrm{N}), 1126(\mathrm{C}-\mathrm{O}-\mathrm{C}) ;{ }^{1} \mathrm{H}$ NMR $\left(\delta ; \mathrm{CDCl}_{3} ; 300 \mathrm{MHz}\right)$ : 8.4-8.3 (m, 4H, H2' e H3'), 7.2 (s, 1H, H4), 6.7 (s, 1H, H6), 6,0-5.9 (m, $\left.1 \mathrm{H}, \mathrm{CH}_{2}=\mathrm{CH}-\mathrm{CH}_{2}\right)$, 5.1-5.1 (m, $2 \mathrm{H}, \mathrm{CH}_{2}=\mathrm{CH}-$ $\left.\mathrm{CH}_{2}\right), 4.0\left(\mathrm{~s}, 3 \mathrm{H}, \mathrm{OC}_{3}\right), 3.5\left(\mathrm{~d}, 2 \mathrm{H}, \mathrm{CH}_{2}=\mathrm{CH}-\overline{\mathrm{CH}}_{2} ; J=\right.$ $6.6 \mathrm{~Hz}) ;{ }^{13} \mathrm{C}$ NMR $\left(\delta ; \mathrm{CDCl}_{3} ; 75 \mathrm{MHz}\right): 160.6(\mathrm{C} 2), 149.2$ (C4'), 144.5 (C7), 143.6 (C3a), 138.9 (C5), $138.3\left(\mathrm{Cl}^{\prime}\right), 137.0$ $\left(\mathrm{CH}_{2}=\underline{\mathrm{CH}}-\mathrm{CH}_{2}\right), 132.6(\mathrm{C} 7 \mathrm{a}), 128.3\left(\mathrm{C}^{\prime}\right), 124.0\left(\mathrm{C}^{\prime}\right), 116.3$ $\left(\mathrm{CH}_{2}=\overline{\mathrm{C}} \mathrm{H}-\mathrm{CH}_{2}\right), 112.3(\mathrm{C} 4), 109.5(\mathrm{C} 6), 56.3\left(\mathrm{OCH}_{3}\right), 40.4$ $\left(\mathrm{CH}_{2}=\mathrm{CH}-\mathrm{CH}_{2}\right)$. HRMS-ESI: $m / z$ calcd for $\mathrm{C}_{17} \mathrm{H}_{14} \mathrm{O}_{4} \mathrm{~N}_{2}$ $(\mathrm{M}+\mathrm{H})^{+}$: 311.1026; found: 311.1024.

7-Methoxy-2-phenyl-5-propylbenzo[d]oxazol $\left(\mathbf{5} \boldsymbol{a}^{\prime}\right)$. Yellow semi-solid; $27 \%$ yield; Rf 0.79 ; IR $\left(\bar{v}_{\text {máx }}\right.$, in $\left.\mathrm{cm}^{-1}\right): 1623(\mathrm{C}=\mathrm{N})$, 1601, 1496, 1465 (C=C), 1309, 1199 (C-N), 1129 (C-O-C); ${ }^{1} \mathrm{H}$ NMR $\left(\delta ; \mathrm{CDCl}_{3} ; 300 \mathrm{MHz}\right): 8.2-8.2\left(\mathrm{~m}, 2 \mathrm{H}, \mathrm{H}^{\prime}\right)$, 7.5-7.4 $\left(\mathrm{m}, 3 \mathrm{H}, \mathrm{H}^{\prime}\right.$ e H4 $\left.4^{\prime}\right), 7.21-7.02(\mathrm{~m}, 1 \mathrm{H}, \mathrm{H} 4), 6.7(\mathrm{~d}, 1 \mathrm{H}, \mathrm{H} 6$; $J=1.2 \mathrm{~Hz}), 4.0\left(\mathrm{~s}, 3 \mathrm{H}, \mathrm{OCH}_{3}\right), 2.6\left(\mathrm{t}, 2 \mathrm{H}, \mathrm{CH}_{3}-\mathrm{CH}_{2}-\mathrm{CH}_{2} ;\right.$ $J=7.2 \mathrm{~Hz}), 1.7-1.6\left(\mathrm{~m}, 2 \mathrm{H}, \mathrm{CH}_{3}-\mathrm{CH}_{2}-\mathrm{CH}_{2}\right), 0.9(\mathrm{t}, 3 \overline{\mathrm{H}}$, $\left.\mathrm{C}_{\underline{3}}-\mathrm{CH}_{2}-\mathrm{CH}_{2} ; J=7.2 \mathrm{~Hz}\right) ;{ }^{13} \mathrm{C} \mathrm{NMR}\left(\delta ; \mathrm{CDCl}_{3} ; 75 \mathrm{MHz}\right)$ : 162.9 (C2), 144.1 (C7), 143.8 (C3a), 140.2 (C5), 131.3 (C4'), $128.7\left(\mathrm{C} 3^{\prime}\right), 128.4(\mathrm{C} 7 \mathrm{a}), 127.5\left(\mathrm{C}^{\prime}\right), 127.1\left(\mathrm{Cl}^{\prime}\right), 111.6(\mathrm{C} 6)$, 108.8 (C4), $56.3\left(\mathrm{OCH}_{3}\right), 38.4\left(\mathrm{CH}_{3}-\mathrm{CH}_{2}-\mathrm{CH}_{2}\right), 24.9\left(\mathrm{CH}_{3}\right.$ $\left.\mathrm{CH}_{2}-\mathrm{CH}_{2}\right), 13.7\left(\mathrm{CH}_{3}-\mathrm{CH}_{2}-\mathrm{CH}_{2}\right)$. HRMS-ESI: $\mathrm{m} / z$ calcd for $\overline{\mathrm{C}}_{17} \mathrm{H}_{17} \mathrm{O}_{2} \mathrm{~N}(\mathrm{M}+\overline{\mathrm{H}})^{+}: 268.1332$; found: 268.1338 .

2-(4-Chlorophenyl)-7-methoxy-5-propylbenzo[d]oxazol (5 $\left.\boldsymbol{b}^{\prime}\right)$. Light brown crystals; $26 \%$ yield; m.p.: $81.5-84.5^{\circ} \mathrm{C}$; Rf 0.81 ; IR $\left(\bar{v}_{\text {máx }}\right.$, in $\left.\mathrm{cm}^{-1}\right): 1626(\mathrm{C}=\mathrm{N}), 1596,1577,1493,1456$ $(\mathrm{C}=\mathrm{C}), 1319,1201(\mathrm{C}-\mathrm{N}), 1132(\mathrm{C}-\mathrm{O}-\mathrm{C}) ;{ }^{1} \mathrm{H}$ NMR $\left(\delta ; \mathrm{CDCl}_{3}\right.$; $300 \mathrm{MHz}): 8.2-8.1\left(\mathrm{~m}, 2 \mathrm{H}, \mathrm{H}^{\prime}\right)$ ), 7.5-7.4 (m, 2H, H3' $), 7.2-7.0$ (m, 1H, H4), $6.7(\mathrm{~d}, 1 \mathrm{H}, \mathrm{H} 6 ; J=1.2 \mathrm{~Hz}), 4.0\left(\mathrm{~s}, 3 \mathrm{H}, \mathrm{OC}_{3}\right)$, $2.6\left(\mathrm{t}, 2 \mathrm{H}, \mathrm{CH}_{3}-\mathrm{CH}_{2}-\mathrm{CH}_{2} ; J=7.2 \mathrm{~Hz}\right), 1.7-1.6\left(\mathrm{~m}, 2 \mathrm{H}, \mathrm{CH}_{3}-\right.$ $\left.\mathrm{CH}_{2}-\mathrm{CH}_{2}\right), 0.9\left(\mathrm{t}, 3 \mathrm{H}, \underline{\mathrm{CH}}_{3}-\mathrm{CH}_{2}-\mathrm{CH}_{2} ; J=7.2 \mathrm{~Hz}\right) ;{ }^{13} \mathrm{C} \mathrm{NMR}$ $\left(\delta ; \overline{\mathrm{CDCl}}_{3} ; 75 \mathrm{MHz}\right): 161,9(\mathrm{C} 2), 144.2(\mathrm{C} 7), 143.6$ (C3a), 140.4 (C5), 137.5 (C7a), $129.1\left(\mathrm{C}^{\prime}\right), 128.8\left(\mathrm{C} 2^{\prime}\right), 125.6\left(\mathrm{C}^{\prime}\right), 121.7$ $\left(\mathrm{Cl}^{\prime}\right), 111.7$ (C6), $108.9(\mathrm{C} 4), 56.3\left(\mathrm{OCH}_{3}\right), 38.4\left(\mathrm{CH}_{3}-\mathrm{CH}_{2}-\right.$ $\left.\mathrm{CH}_{2}\right), 24.9\left(\mathrm{CH}_{3}-\underline{\mathrm{CH}}_{2}-\mathrm{CH}_{2}\right), 13.7\left(\mathrm{C}_{3}-\mathrm{CH}_{2}-\mathrm{CH}_{2}\right)$. HRMSESI: $m / z$ calcd for $\mathrm{C}_{17} \mathrm{H}_{16} \mathrm{O}_{2} \mathrm{NCl}(\mathrm{M}+\mathrm{H})^{+}: 302.0942$; found: 302.0944 .

7-Methoxy-2-(4-methoxyphenyl)-5-propylbenzo[d]oxazol $\left(5 c^{\prime}\right)$. Brown crystals; $30 \%$ yield; m.p.: $89.4-92.5^{\circ} \mathrm{C}$; Rf 0.59; IR $\left(\bar{v}_{\text {máx }}\right.$, in $\left.\mathrm{cm}^{-1}\right): 1622(\mathrm{C}=\mathrm{N}), 1605,1583,1496(\mathrm{C}=\mathrm{C}), 1324$, $1255(\mathrm{C}-\mathrm{N}), 1132(\mathrm{C}-\mathrm{O}-\mathrm{C}) ;{ }^{1} \mathrm{H}$ NMR $\left(\delta ; \mathrm{CDCl}_{3} ; 300 \mathrm{MHz}\right)$ : 8.2-8.1 (m, 2H, H2'), 7.22-7.1 (m, 1H, H4), 7.0-6.9 (m, 2H, $\left.\mathrm{H}^{\prime}\right), 6.6(\mathrm{~d}, 1 \mathrm{H}, \mathrm{H} 6 ; J=1.2 \mathrm{~Hz}), 4.0\left(\mathrm{~s}, 3 \mathrm{H}, \mathrm{C} 7-\mathrm{OCH}_{3}\right), 3.8$ (s, $\left.3 \mathrm{H}, \mathrm{C}^{\prime}-\mathrm{OCH}_{3}\right), 2.6$ (t, $2 \mathrm{H}, \mathrm{CH}_{3}-\mathrm{CH}_{2}-\mathrm{CH}_{2} ; J=7.5 \mathrm{~Hz}$ ), 1.7-1.6 (m, $\left.2 \mathrm{H}, \mathrm{CH}_{3}-\mathrm{CH}_{2}-\mathrm{CH}_{2}\right), 0.9$ (t, $3 \mathrm{H}, \underline{\mathrm{C}}_{3}-\mathrm{CH}_{2}-\mathrm{CH}_{2}$; $J=7.5 \mathrm{~Hz}) ;{ }^{13} \mathrm{C}$ NMR $\left(\delta ; \mathrm{CDCl}_{3} ; 75 \mathrm{MHz}\right): 163.0(\mathrm{C} 2), 162.1$ 
(C4'), 144.0 (C7), 143.9 (C3a), 140.0 (C5), 138.0 (C7a), 129.3 $\left(\mathrm{C}^{\prime}\right), 119.7\left(\mathrm{Cl}^{\prime}\right), 114.2\left(\mathrm{C}^{\prime}\right), 111.4$ (C6), $108.4(\mathrm{C} 4), 56.3$ $\left(\mathrm{C} 7-\mathrm{OCH}_{3}\right), 55.4\left(\mathrm{C}^{\prime}-\mathrm{OCH}_{3}\right), 38.4\left(\mathrm{CH}_{3}-\mathrm{CH}_{2}-\mathrm{CH}_{2}\right), 24.9$ $\left(\mathrm{CH}_{3}-\mathrm{CH}_{2}-\mathrm{CH}_{2}\right), 13.7\left(\mathrm{CH}_{3}-\mathrm{CH}_{2}-\mathrm{CH}_{2}\right)$. HRMS-ESI: $m / z$ calcd for $\mathrm{C}_{18} \mathrm{H}_{19} \mathrm{O}_{3} \mathrm{~N}(\mathrm{M}+\mathrm{H})^{+}$: 298.1438; found: 298.1450 .

7-Methoxy-2-(4-nitrophenyl)-5-propylbenzo[d]oxazol (5d'). Yellow crystals; $33 \%$ yield; m.p.: $136.5-140^{\circ} \mathrm{C}$; Rf 0.72 ; IR $\left(\bar{v}_{\text {máx }}\right.$, in $\left.\mathrm{cm}^{-1}\right): 1625(\mathrm{C}=\mathrm{N}), 1602(\mathrm{C}=\mathrm{C}), 1518,1339\left(\mathrm{NO}_{2}\right)$, 1309, $1200(\mathrm{C}-\mathrm{N}), 1132(\mathrm{C}-\mathrm{O}-\mathrm{C}) ;{ }^{1} \mathrm{H}$ NMR $\left(\delta ; \mathrm{CDCl}_{3}\right.$; $300 \mathrm{MHz}$ ): $8.4-8.3\left(\mathrm{~m}, 4 \mathrm{H}, \mathrm{H} 2^{\prime}\right.$ e $\left.\mathrm{H}^{\prime}\right), 7.2$ (s, $\left.1 \mathrm{H}, \mathrm{H} 4\right), 6.7$ (s, 1H, H6), $4.0\left(\mathrm{~s}, 3 \mathrm{H}, \mathrm{OC}_{3}\right), 2.7\left(\mathrm{t}, 2 \mathrm{H}, \mathrm{CH}_{3}-\mathrm{CH}_{2}-\mathrm{CH}_{2}\right.$; $J=7.5 \mathrm{~Hz}), 1.7-1.6\left(\mathrm{~m}, 2 \mathrm{H}, \mathrm{CH}_{3}-\mathrm{CH}_{2}-\mathrm{CH}_{2}\right), 0.9(\mathrm{t}, 3 \overline{\mathrm{H}}$, $\left.\mathrm{CH}_{3}-\mathrm{CH}_{2}-\mathrm{CH}_{2} ; J=7.5 \mathrm{~Hz}\right) ;{ }^{13} \mathrm{C} \mathrm{NMR}\left(\delta ; \mathrm{CDCl}_{3} ; 75 \mathrm{MHz}\right)$ : 160.5 (C2), $149.2\left(\mathrm{C}^{\prime}\right), 144.3$ (C7), 143.5 (C3a), $141.0\left(\mathrm{Cl}^{\prime}\right)$, 138.7 (C5), 132.7 (C7a), $128.3\left(\mathrm{C}^{\prime}\right), 124.1\left(\mathrm{C}^{\prime}\right), 112.3$ (C6), 109.6 (C4), $56.3\left(\mathrm{OCH}_{3}\right), 38.4\left(\mathrm{CH}_{3}-\mathrm{CH}_{2}-\mathrm{CH}_{2}\right), 24.8\left(\mathrm{CH}_{3}-\right.$ $\left.\mathrm{CH}_{2}-\mathrm{CH}_{2}\right), 13.7\left(\underline{\mathrm{CH}}_{3}-\mathrm{CH}_{2}-\mathrm{CH}_{2}\right)$. HRMS-ESI: $m / z$ calcd for $\overline{\mathrm{C}}_{17} \mathrm{H}_{16} \mathrm{O}_{4} \mathrm{~N}_{2}(\mathrm{M}+\mathrm{H})^{+}$: 313.1183 ; found: 313.1182 .

2.2. Antifungal Activity Evaluation. The antifungal activity evaluation was performed through a Mueller-Hinton broth microdilution method and with the methodology and interpretative criteria proposed by document M27A3 [15]. The stock solutions of all the compounds were prepared in DMSO $1 \%$ at final concentration and tested at concentrations $(\mu \mathrm{g} / \mathrm{mL}) 100 ; 60 ; 30 ; 15 ; 7.5 ; 3.75 ; 1.875 ; 0.468 ; 0.23$; 0.06 . The standard drug fluconazole was applied as control of fungistatic action. Results were visualized and analyzed at $530 \mathrm{~nm}$ in an Anthos Zenyth 200rt Microplate Reader. The inhibitory concentrations of microbial growth were determined at $50 \%\left(\mathrm{IC}_{50}\right)$ and $90 \%\left(\mathrm{IC}_{90}\right)$ in $\mu \mathrm{mol} / \mathrm{mL}$ and compared among the microorganisms. The tests were all done in duplicate.

2.3. Cytotoxicity Assay. The cytotoxicity of the compounds $(200-1.5 \mu \mathrm{g} / \mathrm{mL})$ to peripheral blood mononuclear cells (PBMCs) was determined using the 3-(4,5-dimethylthiazol2-yl)-2,5-diphenyl tetrazolium bromide (MTT) method. The PBMCs were obtained from healthy volunteers by FicollHypaque density gradient centrifugation. These cells were grown in RMPI medium (Cultilab, Brazil) supplemented with $10 \%$ (v/v) heat-inactivated fetal bovine serum (FBS) (Cultilab, Brazil), $100 \mathrm{mg} / \mathrm{mL}$ penicillin, and $100 \mathrm{mg} / \mathrm{mL}$ streptomycin in $5 \% \mathrm{CO} 2$ atmosphere at $37^{\circ} \mathrm{C}$. The cell suspension of PBMCs at a concentration of $2.4 \times 106$ cells $/ \mathrm{mL}$ was distributed in a 96-well plate, $90 \mu \mathrm{L}$ in each well with $10 \mu \mathrm{L}$ of test compounds at different concentrations, and incubated at $37^{\circ} \mathrm{C}$ in an incubator at $5 \% \mathrm{CO} 2$ for $48 \mathrm{~h}$. After that, $10 \mu \mathrm{L}$ of MTT dye was added $(5 \mathrm{mg} / \mathrm{mL})$ and the cells were incubated again for an additional $4 \mathrm{~h}$ period. Then, the medium was carefully removed and $100 \mu \mathrm{L}$ of DMSO was added for solubilization of formazan crystals. The plates were shaken for $5 \mathrm{~min}$, and absorbance for each sample was measured in a spectrophotometric microplate reader at $560 \mathrm{~nm}$. The percentage of cytotoxicity was calculated as $[(A-B) / A \times 100]$, where $A$ and $B$ are the absorbance of control and treated cells, respectively. Data were analyzed using linear regression to obtain values for $\mathrm{CC}_{50}$ and $\mathrm{CC}_{90}$ (cytotoxic concentration for $50 \%$ and $90 \%$ of cells, resp.). Selectivity indexes were expressed as the ratio $\mathrm{CC}_{50} / \mathrm{IC}_{50}$. The tests were all done in duplicate.

\section{Results and Discussion}

3.1. Chemistry. The synthetic route used to obtain the molecular hybrids is depicted in Scheme 1.

The synthesis of hybrid compounds was accomplished initially by orto-nitration of eugenol (1) and dihydroeugenol $\left(\mathbf{1}^{\prime}\right)$ using sodium nitrate, potassium bisulfate, and wet silica gel in dichloromethane, as reported by Zolfigol and coworkers [16]. These nitroderivatives ( 2 and $\left.2^{\prime}\right)$ were obtained in good yields as dark orange oils after successive extractions of the crude material with hexane and solvent evaporation. The next step consisted in reducing these nitrated derivatives to the known aminophenols derivatives $\mathbf{3}$ and $\mathbf{3}^{\prime}$. This objective was achieved by employing the classical and mild method of reduction of nitrocompounds with stannous chloride in ethanol [17] and the amphoteric intermediates were obtained as pale yellow solids after careful acid-base extractions. The data from spectrometric analysis of intermediates 2, 2', 3, and $3^{\prime}$ showed perfect agreement with those found in the literature [18]. Following, these aminophenols were converted to $N$-(o-hydroxyphenyl)benzamides by acylation in mild conditions with appropriate carboxylic acid chlorides in an ice-cooled mixture of ether-water and sodium bicarbonate [19]. The benzamides $\mathbf{4 a - 4 d}$ and $\mathbf{4} \mathbf{a}^{\prime}-\mathbf{4} \mathbf{d}^{\prime}$ were obtained in excellent yields after filtration or solvent extraction. NMR spectra of these intermediates clearly showed the identity of the products and the maintenance of free phenolic hydroxyl, necessary to the next reaction step. Finally, benzoxazoles $\mathbf{5 a}-\mathbf{5} \mathbf{d}$ and $\mathbf{5} \mathbf{a}^{\prime}-\mathbf{5} \mathbf{d}^{\prime}$ were synthesized by adaptation of the methods described by Nakamura et al. [20] and Yang et al. [21] in which the amides previously obtained were heated under reflux in toluene with $p$-toluenesulfonic acid as a catalyst and dehydrating agent. The products were obtained in high yields as single products after filtering the reaction mixture through a short pad of silica gel with dichloromethane and removing the solvent in rotavapor. The eight benzoxazoles are new compounds and were fully characterized by IR, NMR, and HRMS analysis.

3.2. Biological Evaluations. The final eight compounds were screened for their potential antifungal activity against five fungi species: Candida albicans (C. albicans), C. tropicalis, C. krusei, C. parapsilosis, and C. glabrata (Table 1). The results were calculated using the inhibitory concentration of $50 \%$ microbial growth and the interpretative criteria were those proposed by the document M27A3 from the Clinical and Laboratory Standards Institute [15]. Fluconazole was used as the positive control. The assays were performed in triplicate and the results from these replicates were identical.

Our expectation was that the fusion of two pharmacophores, that is, eugenol and benzoxazole ring, would enhance eugenol antimicrobial profiles against those fungi. In fact, four products showed fungistatic properties in the range $321-380 \mu \mathrm{M}$. Moreover, it is interesting to note that these 
TABLE 1: In vitro antifungal activity $\left(\mathrm{IC}_{50}, \mu \mathrm{M}\right)$, cytotoxic activity $\left(\mathrm{CC}_{50}, \mu \mathrm{M}\right)$, and selectivity index for eugenol (1) and related benzoxazole derivatives $\left(5 \mathrm{a}-5 \mathrm{~d}^{\prime}\right)$.

\begin{tabular}{|c|c|c|c|c|c|c|c|}
\hline Compound & $\begin{array}{c}\text { C. albicans } \\
\text { ATCC } 10231\end{array}$ & $\begin{array}{c}\text { C. tropicalis } \\
\text { ATCC } 750\end{array}$ & $\begin{array}{c}\mathrm{IC}_{50}[\mu \mathrm{M}] \\
\text { C. } k r u s e i \\
\text { ATCC } 6258\end{array}$ & $\begin{array}{c}\text { C. parapsilosis } \\
\text { ATCC } 22019\end{array}$ & $\begin{array}{c}\text { C. glabrata } \\
\text { ATCC } 90030\end{array}$ & $\mathrm{CC}_{50}[\mu \mathrm{M}]^{*}$ & SI \\
\hline 1 & 1524 & $-^{\mathrm{a}}$ & 1524 & $-^{\mathrm{a}}$ & 1524 & 360 & 0.2 \\
\hline $5 a$ & 380 & $-^{\mathrm{a}}$ & $-^{a}$ & $-^{a}$ & $-^{a}$ & 1111 & 2.9 \\
\hline $5 a^{\prime}$ & $-^{\mathrm{a}}$ & $-^{\mathrm{a}}$ & $-^{a}$ & $-^{\mathrm{a}}$ & $-^{\mathrm{a}}$ & 489 & $-^{\mathrm{b}}$ \\
\hline $5 b$ & $-{ }^{\mathrm{a}}$ & $-^{\mathrm{a}}$ & $-{ }^{a}$ & $-{ }^{\mathrm{a}}$ & $-^{\mathrm{a}}$ & 558 & $-^{\mathrm{b}}$ \\
\hline $5 b^{\prime}$ & $-^{\mathrm{a}}$ & $-^{\mathrm{a}}$ & 332 & $-^{\mathrm{a}}$ & 332 & 629 & 1.9 \\
\hline $5 c$ & 331 & $-^{\mathrm{a}}$ & $-^{\mathrm{a}}$ & $-^{\mathrm{a}}$ & 338 & 888 & 2.6 \\
\hline $5 c^{\prime}$ & $-^{\mathrm{a}}$ & $-^{\mathrm{a}}$ & $-^{\mathrm{a}}$ & $-^{\mathrm{a}}$ & $-^{\mathrm{a}}$ & 1216 & $-^{b}$ \\
\hline $5 d$ & $-^{\mathrm{a}}$ & $-^{\mathrm{a}}$ & $-{ }^{a}$ & $-^{\mathrm{a}}$ & $-{ }^{\mathrm{a}}$ & 1208 & $-^{\mathrm{b}}$ \\
\hline $5 d^{\prime}$ & 321 & $-^{\mathrm{a}}$ & 321 & $-^{\mathrm{a}}$ & $-^{\mathrm{a}}$ & 613 & 1.9 \\
\hline FLC & 1.6 & 3.3 & 104.3 & 3.3 & 52.2 & $-^{\mathrm{b}}$ & $-^{\mathrm{b}}$ \\
\hline
\end{tabular}

${ }^{*}$ The experiments were done in triplicate and the results were identical; ${ }^{a}$ no significant activity; ${ }^{b}$ not determined; SI: selectivity indexes, expressed as the ratio $\mathrm{CC}_{50} / \mathrm{IC}_{50}$; FLC: fluconazole.<smiles>[R]c1ccc(O)c(OC)c1</smiles>

$1 / 1^{\prime}$

1/2/3: R = allyl $1^{\prime} / 2^{\prime} / 3^{\prime}: \mathrm{R}=$ propyl<smiles>[R]c1cc(OC)c(O)c([N+](=O)[O-])c1</smiles>

$2 / 2^{\prime}$

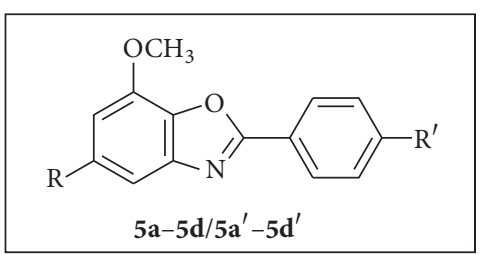

(iv)<smiles>[R]c1cc(N)c(O)c(OC)c1</smiles>

$3 / 3^{\prime}$<smiles>C1CC1</smiles><smiles>[R]c1ccc(C(=O)Nc2cc([R])cc(OC)c2O)cc1</smiles>

$4 a-4 d / 4 a^{\prime}-4 d^{\prime}$

4a/5a: $\mathrm{R}=$ allyl; $\mathrm{R}^{\prime}=\mathrm{H}$

$4 \mathrm{a}^{\prime} / 5 \mathrm{a}^{\prime}: \mathrm{R}=$ propyl; $\mathrm{R}^{\prime}=\mathrm{H}$

$4 \mathbf{b} / 5 \mathbf{b}: \mathrm{R}=$ allyl; $\mathrm{R}^{\prime}=\mathrm{Cl}$

$4 \mathbf{b}^{\prime} / 5 \mathbf{b}^{\prime}: \mathrm{R}=$ propyl; $\mathrm{R}^{\prime}=\mathrm{Cl}$
$4 \mathrm{c} / 5 \mathrm{c}: \mathrm{R}=$ allyl; $\mathrm{R}^{\prime}=\mathrm{OCH}_{3}$

$4 \mathrm{c}^{\prime} / 5 \mathrm{c}^{\prime}: \mathrm{R}=$ propyl; $\mathrm{R}^{\prime}=\mathrm{OCH}_{3}$

4d/5d: $\mathrm{R}=$ allyl; $\mathrm{R}^{\prime}=\mathrm{NO}_{2}$

$4 d^{\prime} / 5 d^{\prime}: \mathrm{R}=$ propyl; $\mathrm{R}^{\prime}=\mathrm{NO}_{2}$

Scheme 1: Synthetic route to derivatives $\mathbf{5 a}-\mathbf{5 d}$ and $\mathbf{5} \mathbf{a}^{\prime} \mathbf{-} \mathbf{5} \mathbf{d}^{\prime}$. (i) $\mathrm{NaNO}_{3}, \mathrm{KHSO}_{4}$, silica/water (1:1, w/w), dichloromethane, r.t.; (ii) $\mathrm{SnCl}_{2} \cdot 2 \mathrm{H}_{2} \mathrm{O}$, ethanol, $70^{\circ} \mathrm{C}$; (iii) benzoyl chlorides, $\mathrm{NaHCO}_{3}$, ethyl ether/water $(9: 1, \mathrm{v} / \mathrm{v}), 0^{\circ} \mathrm{C}$; (iv) PTSA, toluene, reflux.

derivatives were about five times more potent than eugenol, which in turn could only inhibit three strains growing up to the highest concentration used. Benzoxazoles $\mathbf{5 a}, \mathbf{5 c}$, and $\mathbf{5} \mathbf{d}^{\prime}$ showed good fungistatic activities against C. albicans ( $\mathrm{IC}_{50}$ values of 380,331 , and $321 \mu \mathrm{M}$, resp.), while compounds $\mathbf{5} \mathbf{b}^{\prime}$ and $\mathbf{5 c}$ could also inhibit C. glabrata growing at 332 and $338 \mu \mathrm{M}$, respectively. Derivatives $\mathbf{5} \mathbf{b}^{\prime}$ and $\mathbf{5} \mathbf{d}^{\prime}$ inhibited also C. krusei growing at $\mathrm{IC}_{50} 332$ and $321 \mu \mathrm{M}$, sequentially.
Notably, this shows that the potential of this new kind of derivative against this strain since C. krusei is naturally resistant to many azole drugs, for example, fluconazole [22]. Our study corroborates the findings of Carrasco et al. [23], which showed that the phenolic hydroxyl was not necessary in antifungal action against Candida sp. An influence of the substituent group on the para-position of phenyl ring was observed for products $\mathbf{5 c}$ and $\mathbf{5 d}$ since the presence 
of the electron-donating group in $\mathbf{5 c}$ led to an activated benzoxazole, while the nitrosubstituted product $\mathbf{5 d}$ is among the inactive compounds. However, it is not possible to use this observation as a rule, since, in propyl derivatives $\mathbf{5} \mathrm{c}^{\prime}$ and $\mathbf{5} \mathbf{d}^{\prime}$, one can see the opposite.

The selective toxicity of these benzoxazoles, expressed as the selectivity index (SI), was then assessed on peripheral human blood mononuclear cells. This parameter reflects the quantity of compound that is active against the pathogen but is not toxic towards the host cell. Therefore, compounds with a higher SI are very promising because the concentration of the compound to induce an antimicrobial activity is lower than the dose that induces cytotoxicity in host cells. The analysis of SI values is very important to establish if the chemical modifications in each of the compounds can increase the antifungal activity and decrease the cytotoxicity to host cells.

The results from cytotoxicity assays showed that all hybrids had higher $\mathrm{CC}_{50}$ values than eugenol (Table 1). Compounds $\mathbf{5 a}, \mathbf{5} \mathbf{b}^{\prime}, \mathbf{5} \mathbf{c}$, and $\mathbf{5} \mathbf{d}^{\prime}$ presented selectivity index (SI) values 9 to 15 times higher than eugenol. The blockade of the phenolic hydroxyl may be related to the decreased toxicity of eugenol in mammalian cells, while the nature of para-substituents in phenyl ring showed no clear relationship with the observed cytotoxicity profile, as noted in the results of antifungal evaluation. Meantime, it could be noted that derivatives $\mathbf{5} \mathbf{a}$ and $\mathbf{5} \mathbf{d}$ were less toxic than their propyl analogs $5 \mathbf{a}^{\prime}$ and $\mathbf{5} \mathbf{d}^{\prime}$. In vivo, the allyl group can lead to toxic metabolic products [24], but it is not always a toxicophore, since this will depend on other structural factors associated with the whole molecule.

In short, these are new antifungal prototypes, which may be used for future chemical modifications to improve activity and reduce cytotoxicity.

\section{Conclusion}

The present study described the synthesis and antifungal evaluation of benzoxazoles devised by molecular hybridization with eugenol or dihydroeugenol. Four of them (5a, $5 \mathbf{b}^{\prime}, \mathbf{5} \mathbf{c}$, and $5 \mathbf{c}^{\prime}$ ) showed activity against pathogenic and opportunistic species of Candida spp. against which eugenol had only modest activity. Furthermore, they were 9-15 times more selective than eugenol in cytotoxicity test. The ongoing investigation of the mechanism by which these substances play antifungal effects can lead to a new class of antimicrobial candidates and these benzoxazoles can be submitted to structural variation for optimization of antifungal activity.

\section{Conflicts of Interest}

The authors declare that there are no conflicts of interest regarding the publication of this paper.

\section{Acknowledgments}

The authors acknowledge FAPEMIG for financial support (APQ-01268-16 and APQ-01209-13) and CNPq for the scholarship (Process 130241/2014-8).

\section{References}

[1] P. N. Fonkwo, "Pricing infectious disease. The economic and health implications of infectious diseases," EMBO Reports, vol. 9, supplement 1, pp. S13-S17, 2008.

[2] N. Chami, F. Chami, S. Bennis, J. Trouillas, and A. Remmal, "Antifungal treatment with carvacrol and eugenol of oral candidiasis in immunosuppressed rats," Brazilian Journal of Infectious Diseases, vol. 8, pp. 217-226, 2004.

[3] P. Martins, J. Jesus, S. Santos et al., "Heterocyclic anticancer compounds: Recent advances and the paradigm shift towards the use of nanomedicine's tool Box," Molecules, vol. 20, no. 9, pp. 16852-16891, 2015.

[4] A. Majumder, R. Gupta, and A. Jain, "Microwave-assisted synthesis of nitrogen-containing heterocycles," Green Chemistry Letters and Reviews, vol. 6, no. 2, pp. 151-182, 2013.

[5] T. Ertan, I. Yildiz, B. Tekiner-Gulbas et al., "Synthesis, biological evaluation and 2D-QSAR analysis of benzoxazoles as antimicrobial agents," European Journal of Medicinal Chemistry, vol. 44, no. 2, pp. 501-510, 2009.

[6] M. Medebielle, S. Ait-Mohand, C. Burkhloder, W. R. Jr. Dolbier, G. Laumond, and A. M. Aubertin, "Synthesis of new difluoromethylene benzoxazole and 1,2,4-oxadiazole derivatives, as potent non-nucleoside HIV-1 reverse transcriptase inhibitors," Journal of Fluorine Chemistry, vol. 126, pp. 535-542, 2005.

[7] P. K. Jauhari, A. Bhavani, S. Varalwar, K. Singhal, and P. Raj, "Synthesis of some novel 2-substituted benzoxazoles as anticancer, antifungal, and antimicrobial agents," Medicinal Chemistry Research, vol. 17, no. 2-7, pp. 412-424, 2008.

[8] M. Arisoy, O. Temiz-Arpaci, I. Yildiz et al., "Synthesis, antimicrobial activity and QSAR studies of 2,5-disubstituted benzoxazoles," SAR and QSAR in Environmental Research, vol. 19, no. 5-6, pp. 589-612, 2008.

[9] B. J. Kim, J. Kim, Y. K. Kim, S. Y. Choi, and H. Y. P. Choo, "Synthesis of benzoxazole amides as novel antifungal agents against Malassezia furfur," Bulletin of the Korean Chemical Society, vol. 31, pp. 1270-1274, 2010.

[10] J.-I. Kuroyanagi, K. Kanai, Y. Sugimoto et al., "1,3-Benzoxazole4-carbonitrile as a novel antifungal scaffold of $\beta$-1,6-glucan synthesis inhibitors," Bioorganic and Medicinal Chemistry, vol. 18, no. 21, pp. 7593-7606, 2010.

[11] T. S. Kaufman, "The multiple faces of Eugenol. A versatile starting material and building block for organic and bio-organic synthesis and a convenient precursor toward bio-based fine chemicals," Journal of the Brazilian Chemical Society, vol. 26, no. 6, pp. 1055-1085, 2015.

[12] R. di Pasqua, N. Hoskins, G. Betts, and G. Mauriello, "Changes in membrane fatty acids composition of microbial cells induced by addiction of thymol, carvacrol, limonene, cinnamaldehyde, and eugenol in the growing media," Journal of Agricultural and Food Chemistry, vol. 54, no. 7, pp. 2745-2749, 2006.

[13] G. B. Zore, A. D. Thakre, S. Jadhav, and S. M. Karuppayil, "Terpenoids inhibit Candida albicans growth by affecting membrane integrity and arrest of cell cycle," Phytomedicine, vol. 18, no. 13, pp. 1181-1190, 2011.

[14] A. O. Gill and R. A. Holley, "Mechanisms of bactericidal action of cinnamaldehyde against Listeria monocytogenes and of eugenol against L. monocytogenes and Lactobacillus sakei," Applied and Environmental Microbiology, vol. 70, no. 10, pp. 5750-5755, 2004.

[15] Clinical and Laboratory Standards Institute (CLSI), Reference Method for Broth Dilution Antifungal Susceptibility Testing of 
Yeasts, Approved Standard-, M27-A3, CLSI, Wayne, PA, USA, 3rd edition, 2008.

[16] M. A. Zolfigol, E. Ghaemi, and E. Madrakian, "Nitration of phenols under mild and heterogeneous conditions," Molecules, vol. 6, no. 7, pp. 614-620, 2001.

[17] F. D. Bellamy and K. Ou, "Selective reduction of aromatic nitro compounds with stannous chloride in non acidic and non aqueous medium," Tetrahedron Letters, vol. 25, no. 8, pp. 839$842,1984$.

[18] D. E. Levin and A. Lowy, "Derivatives of dihydroeugenol and certain pharmacological properties of some of the compounds," Journal of the American Chemical Society, vol. 55, no. 5, pp. 19952000, 1933.

[19] E. A. Sener, K. K. Bingöl, I. Ören, Ö. T. Arpaci, I. Yalçin, and N. Altanlar, "Synthesis and microbiological activity of some $\mathrm{N}$-(o-hydroxyphenyl)benzamides and phenylacetamides as the possible metabolites of antimicrobial active benzoxazoles: Part II," Farmaco, vol. 55, no. 6-7, pp. 469-476, 2000.

[20] H. Nakamura, Y. Yasui, and H. S. Ban, "Synthesis and biological evaluation of ortho-carborane containing benzoxazole as an inhibitor of hypoxia inducible factor (HIF)-1 transcriptional activity," Journal of Organometallic Chemistry, vol. 747, pp. 189194, 2013.

[21] X. Yang, G. Shan, and Y. Rao, "Synthesis of 2-aminophenols and heterocycles by Ru-catalyzed C-H mono- and dihydroxylation," Organic Letters, vol. 15, no. 10, pp. 2334-2337, 2013.

[22] J. Loeffler and D. A. Stevens, "Antifungal drug resistance," Clinical Infectious Diseases, vol. 36, no. 1, pp. S31-S41, 2003.

[23] H. Carrasco, M. Raimondi, L. Svetaz et al., "Antifungal activity of eugenol analogues. Influence of different substituents and studies on mechanism of action," Molecules, vol. 17, no. 1, pp. 1002-1024, 2012.

[24] L. M. Lima, "Safrole and the versatility of a natural biophore," Revista Virtual de Quimica, vol. 7, no. 2, pp. 495-538, 2015. 

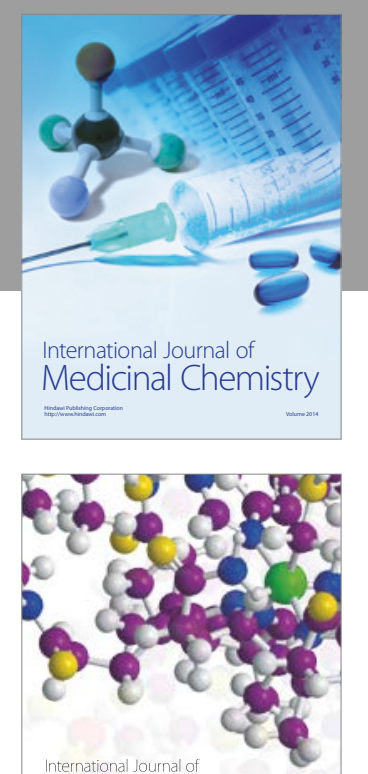

Carbohydrate Chemistry

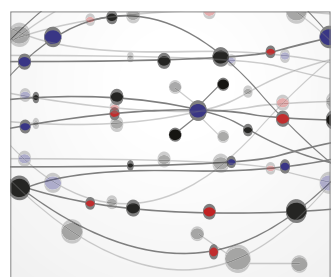

The Scientific World Journal
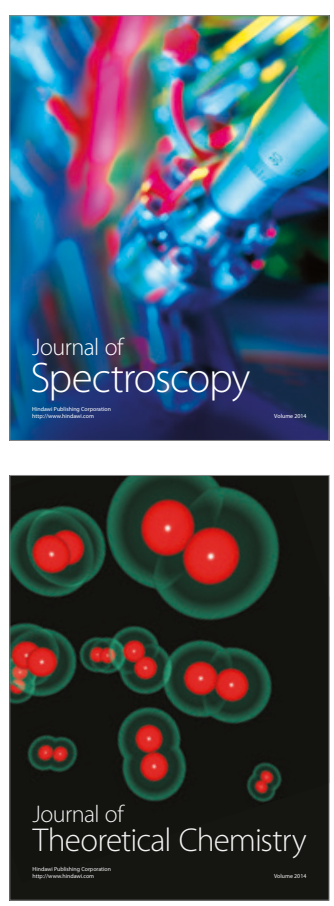
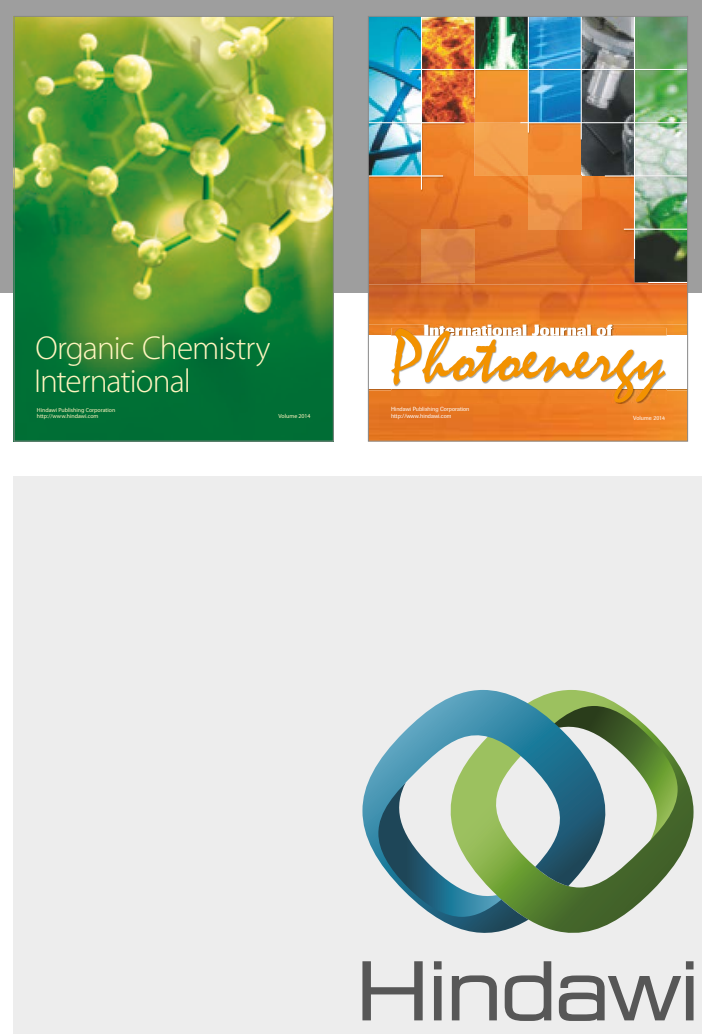

Submit your manuscripts at

https://www.hindawi.com

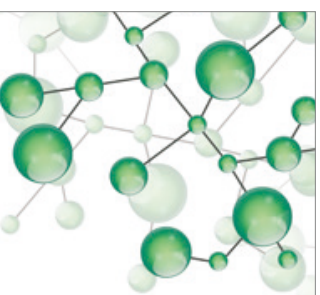

International Journal of

Inorganic Chemistry

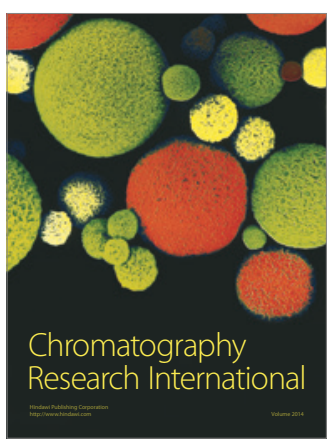

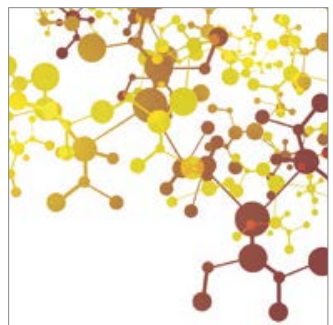

Applied Chemistry
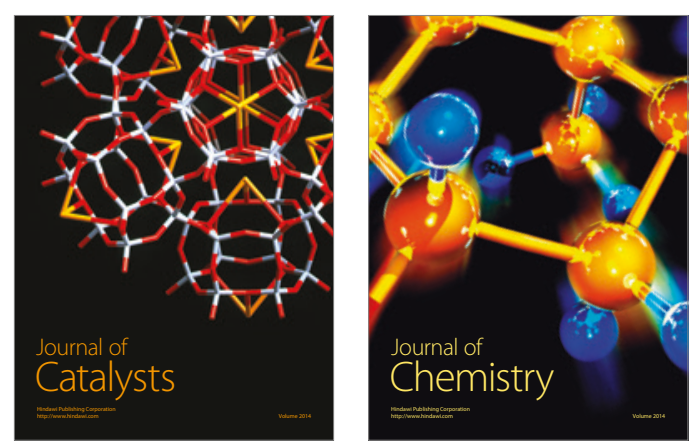
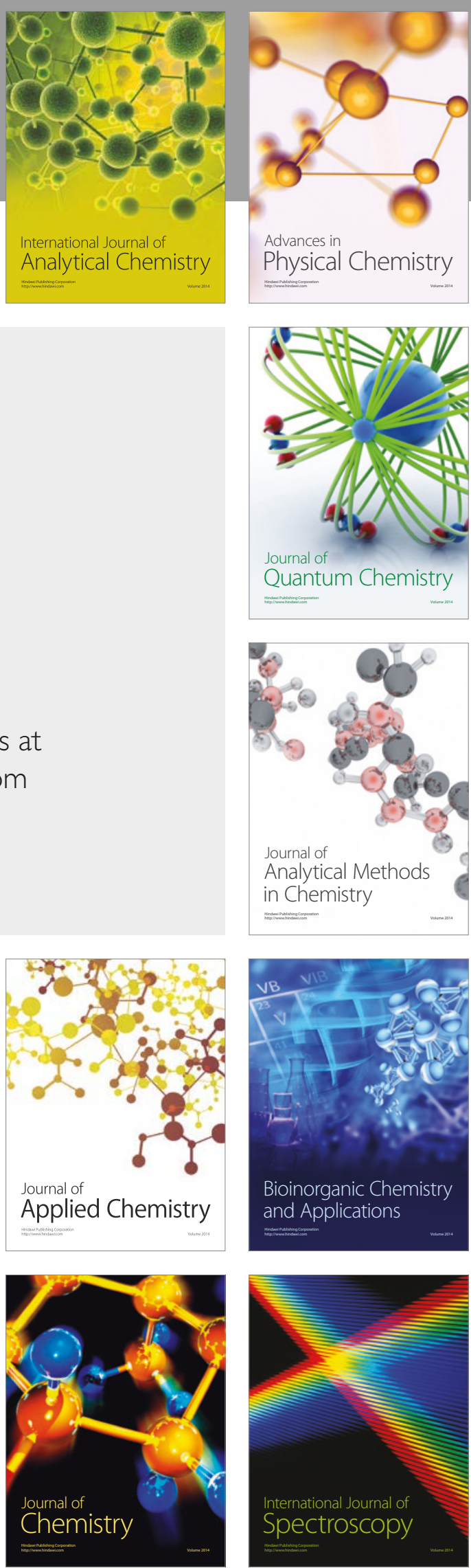